\title{
REVIEWS
}

\section{Post-transcriptional regulation of gene expression in innate immunity}

\section{Susan Carpenter ${ }^{*}$, Emiliano P. Ricci ${ }^{2 *}$, Blandine C. Mercier ${ }^{2 *}$, Melissa J. Moore ${ }^{2}$ and Katherine A. Fitzgerald ${ }^{1,3}$}

Abstract | Innate immune responses combat infectious microorganisms by inducing inflammatory responses, antimicrobial pathways and adaptive immunity. Multiple genes within each of these functional categories are coordinately and temporally regulated in response to distinct external stimuli. The substantial potential of these responses to drive pathological inflammation and tissue damage highlights the need for rigorous control of these responses. Although transcriptional control of inflammatory gene expression has been studied extensively, the importance of post-transcriptional regulation of these processes is less well defined. In this Review, we discuss the regulatory mechanisms that occur at the level of mRNA splicing, mRNA polyadenylation, mRNA stability and protein translation, and that have instrumental roles in controlling both the magnitude and duration of the inflammatory response.

${ }^{1}$ Program in Innate Immunity, Division of Infectious Diseases and Immunology Department of Medicine, University of Massachusetts Medical School, Worcester Massachusetts 01605, USA. ${ }^{2}$ Howard Hughes Medical Institute, University of Massachusetts Medical School, Worcester Massachusetts 01605, USA. ${ }^{3}$ Centre of Molecular Inflammation Research, Department of Cancer Research and Molecular Medicine, Norwegian University of Science and Technology, 7491 Trondheim, Norway.

* These authors contributed equally to this work. Correspondence to K.A.F. e-mail: kate.fitzgerald@ umassmed.edu doi:10.1038/nri3682
A dynamic and coordinately regulated gene expression programme lies at the heart of the inflammatory process. This response endows the host with a first line of defence against infection and the capacity to repair and restore damaged tissues. However, unchecked, prolonged or inappropriately scaled inflammation can be detrimental to the host and lead to diseases such as atherosclerosis, arthritis and cancer ${ }^{1,2}$.

The acute inflammatory programme is initiated when germline-encoded pattern recognition receptors (PRRs) that are present in distinct cellular compartments respond to signs of microbial infection ${ }^{3,4}$. Once activated, these receptors trigger signalling cascades that converge on well-defined transcription factors. Mobilization of these factors leads to rapid, dynamic and temporally regulated changes in the expression of hundreds of genes that are involved in antimicrobial defence, phagocytosis, cell migration, tissue repair and the regulation of adaptive immunity.

Multiple genes within distinct functional categories are coordinately and temporally regulated by transcriptional 'on' and 'off' switches that account for the specificity of gene expression in response to external stimuli. Multiple layers of regulation - including chromatin state, histone or DNA modifications, and the recruitment of transcription factors and of the basal transcription machinery collaborate to control these pathogen-induced or danger signal-induced gene expression programmes ${ }^{5,6}$, which vary depending on the cell lineage involved and the specific signal that is encountered. Although transcription is an essential first step, and certainly the most wellscrutinized area in studies of innate immunity ${ }^{5,6}$, proper regulation of immune genes also involves a plethora of additional post-transcriptional checkpoints. These occur at the level of mRNA splicing, mRNA polyadenylation, mRNA stability and protein translation. Many of these mechanisms are particularly important for modulating the strength and duration of the response and for turning the system off in a timely and efficient manner. In this Review, we cover exciting recent developments in this underexplored area. We also highlight the emerging role of long non-coding RNAs (lncRNAs) in controlling the inflammatory response. A better understanding of these processes could facilitate the development of selective therapeutics to prevent damaging inflammation.

\section{Alternative splicing in innate immunity}

Although transcriptional regulation has been at the forefront of studies of innate immunity, the role of posttranscriptional regulation in controlling gene expression in macrophages and other innate immune cells is equally important. Almost one-fifth of the genes that are expressed in human dendritic cells (DCs) undergo alternative splicing upon bacterial challenge. Most of these genes are involved in general cellular functions but some participate directly in antimicrobial defence ${ }^{7}$. Furthermore, stimulation of human monocytes with the Toll-like receptor 4 (TLR4) ligand lipopolysaccharide 


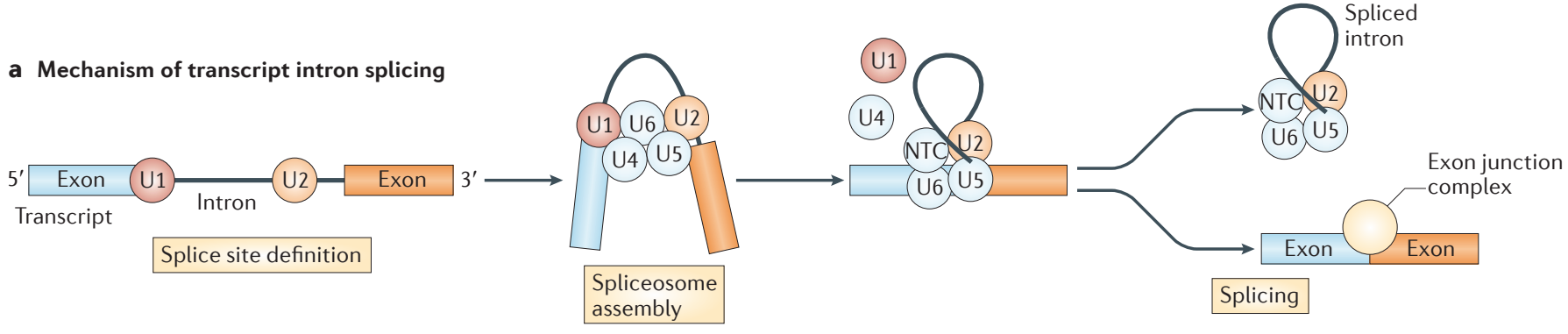

b Mechanism of transcript cleavage and polyadenylation


Figure 1 | Pre-mRNA processing into mature mRNAs: intron splicing and polyadenylation. a | Following transcription, pre-mRNA intronic sequences are removed by splicing. The $5^{\prime}$ and $3^{\prime}$ splice sites of introns are recognized by the small nuclear ribonucleoproteins (snRNPs) U1 and U2, respectively, then the spliceosome assembles and catalyses the excision of the introns and ligation of the flanking exons. A multi-protein complex, the exon junction complex, is deposited on exon-exon junctions. $\mathbf{b} \mid \mathrm{A}$ poly $(\mathrm{A})$ tail is also added to the $3^{\prime}$ end of transcripts. The poly $(\mathrm{A})$ signal and nearby U-rich or GU-rich downstream sequence elements are recognized by two multi-protein complexes — namely, cleavage and polyadenylation specificity factor (CPSF) and cleavage stimulating factor (CSTF), respectively — that promote endonucleolytic cleavage of the transcript. Poly(A) polymerase (PAP) catalyses the subsequent addition of a stretch of adenosines from the cleavage site.

Pattern recognition receptors

(PRRs). Host receptors (such as Toll-like receptors (TLRs) or NOD-like receptors (NLRs)) that can sense pathogenassociated molecular patterns and initiate signalling cascades that lead to an innate immune response. These can be membrane-bound (for example, TLRs) or soluble cytoplasmic receptors (for example, retinoic acidinducible protein I (RIG-I), melanoma differentiationassociated protein 5 (MDA5) and NLRs).

\section{microRNA}

(miRNA). Non-coding RNA (21 nucleotides in length) that is encoded in the genomes of animals and plants. miRNAs regulate gene expression by binding to the $3^{\prime}$ untranslated region of target mRNAs.

AU-rich elements (AREs). Regulatory elements usually located in the 3' untranslated regions of mRNAs that mediate the recognition of an array of RNA-binding proteins and determine

RNA stability and translation.
(LPS) and with interferon- $\gamma($ IFN $\gamma)$ causes the polyadenylation machinery to favour proximal poly(A) site use in terminal exons that contain two or more poly(A) sites $^{8}$. This type of alternative polyadenylation leads to a global shortening of $3^{\prime}$ untranslated regions (UTRs) and a loss of key regulatory elements such as microRNA (miRNA) target sites and AU-rich elements (AREs).

Alternative pre-mRNA processing. Following transcription, pre-mRNA intronic sequences are removed by splicing. The $5^{\prime}$ and $3^{\prime}$ splice sites of introns are recognized by the small nuclear ribonucleic particles (snRNPs) U1 and U2, respectively, before the spliceosome assembles and catalyses excision of the introns and the ligation of flanking exons ${ }^{9}$ (FIG. 1a). In addition, a poly(A) tail is added to the $3^{\prime}$ end of transcripts. A poly(A) signal and nearby U-rich or GU-rich downstream sequence elements (DSEs) are recognized by two multi-protein complexes - namely, cleavage and polyadenylation specificity factor (CPSF) and cleavage stimulation factor (CSTF), respectively - that promote endonucleolytic cleavage of the pre-mRNAs. $\operatorname{Poly}(\mathrm{A})$ polymerase (PAP; also known as PAPa and PAPOLA) subsequently catalyses the addition of a stretch of adenosines from the cleavage $\operatorname{site}^{10}$ (FIG. 1 b).

Remarkably, $>94 \%$ of human genes are subject to alternative splicing and/or alternative polyadenylation ${ }^{11}$. Types of alternative splicing that alter the sequence of the encoded protein include mutually exclusive exons, exon skipping, intron retention and the alternative use of 5' or 3' splice sites at intron ends. Alternative polyadenylation within an intron can also generate an mRNA that encodes a truncated protein product. However, alternative processing is by no means limited to internal sites. Alternative promoter use results in alternative first exons, which changes the length and sequence of the $5^{\prime}$ UTR. Similarly, alternative polyadenylation within the last exon can shorten or extend the $3^{\prime}$ UTR $^{11}$ (FIG. 2a). Modifications to UTRs have important consequences because they can affect sequences that regulate subcellular mRNA localization, translation efficiency and mRNA stability ${ }^{12}$.

Regulation of TLR signalling by alternative splicing and alternative polyadenylation. The TLR signalling pathway is subject to extensive post-transcriptional regulation, in which more than 256 alternatively processed transcripts encode variants of receptors, adaptors and signalling molecules ${ }^{13}$. Every $T L R$ gene has numerous alternatively spliced variants ${ }^{13-18}$, and TLR1 to TLR7 all have between two and four predicted alternative polyadenylation sites ${ }^{16}$. These variant transcripts have myriad effects on signal transduction. For example, an alternatively spliced form of mouse Tlr4 mRNA includes an exon that is not present in the canonical mRNA ${ }^{15}$. An in-frame stop codon in this extra exon generates a secretable receptor isoform that lacks the transmembrane and intracellular domains that are present in the full-length protein. LPS stimulation enhances the expression of soluble TLR4 (smTLR4) by macrophages, and forced overexpression of smTLR4 


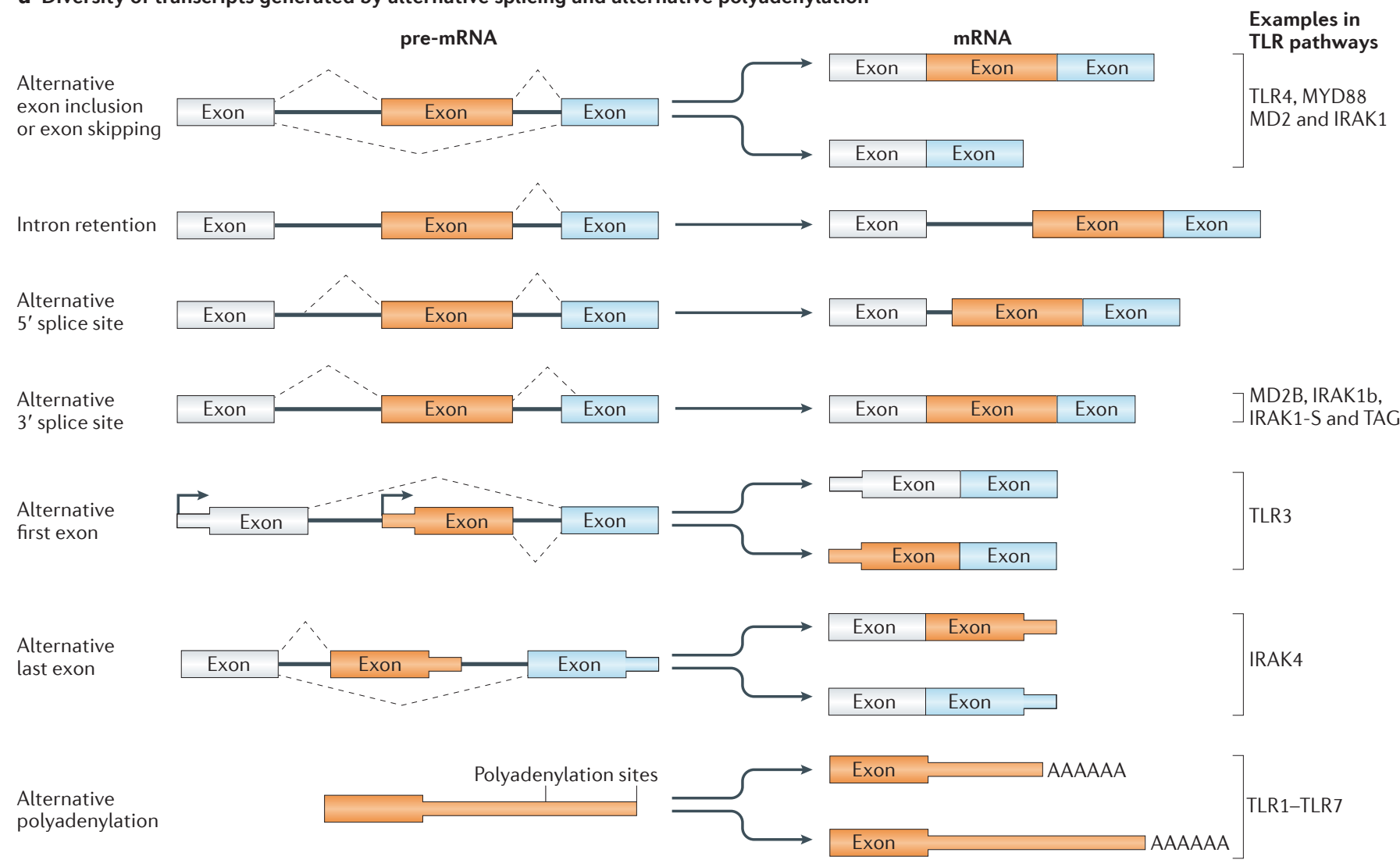

b Regulation of TLR4 signalling by alternative splicing



inhibits LPS-mediated activation of nuclear factor- $\mathrm{kB}$ $(\mathrm{NF}-\mathrm{kB})$ and the production of tumour necrosis factor $(\mathrm{TNF})^{15}$. An analogous TLR4 mRNA isoform that contains a premature stop codon is upregulated following LPS stimulation of human monocytes ${ }^{14}$. Induction of this isoform is significantly lower in monocytes from patients with cystic fibrosis who, compared with healthy controls, produce more TNF in response to LPS ${ }^{14}$. These results suggest that production of a truncated form of TLR4
Figure 2 | Regulation of Toll-like receptor signalling by alternative pre-mRNA processing. a | Toll-like receptor (TLR) signalling pathways are regulated through diverse transcripts that are generated by alternative splicing and alternative polyadenylation. Dashed lines indicate spliced transcript. b| The TLR4 signalling pathway is markedly regulated by alternative splicing of mRNAs encoding the receptor (TLR4) and the co-receptor (MD2), the adaptor molecules (myeloid differentiation primary response protein 88 (MYD88) and TRIF-related adaptor molecule (TRAM)), as well as the IL-1R-associated kinases (IRAKs). Inhibitory isoforms are shown in red. AP-1, activator protein 1; IRF, interferonregulatory factor; LPS, lipopolysaccharide; MD2B, splice variant of MD2; MYD88s, splice variant of MYD88; NF- $\kappa B$, nuclear factor- $\kappa B$; smTLR4, soluble TLR4 splice variant; TAG, splice variant of TRAM; TRAF, TNF receptorassociated factor; TRIF, TIR-domain-containing adaptor protein inducing IFN $\beta$. Part a from REF. 11, Nature Publishing Group.

generates a negative feedback loop that limits excessive inflammation. Another component of this negative feedback mechanism is the requisite TLR4 co-factor MD2 (which is encoded by LY96). Shortened MD2 isoforms have been described in both mouse macrophages ${ }^{19}$ and human monocytic cell lines ${ }^{20}$. The mRNA encoding the mouse MD2B variant lacks the first 54 bases of exon 3 (REF. 19), whereas the mRNA encoding the human MD2s variant lacks all of exon 2 (REF. 20). MD2s expression is 
upregulated by LPS, as well as by IFN $\gamma$ and interleukin- 6 $\left(\right.$ IL-6) ${ }^{20}$. Both MD2B and MD2s proteins bind TLR4 as efficiently as full-length MD2 but they fail to mediate signalling. MD2B inhibits cell surface expression of mouse TLR4 (REF. 19), and MD2s inhibits the binding of full-length MD2 to TLR4 (REF. 20). Thus, these shortened forms of MD2 inhibit macrophage stimulation by LPS ${ }^{19,20}$ by limiting productive interactions with full-length MD2. Together, these results suggest that the production of altered forms of either TLR4 or MD2 modulate macrophage responses to LPS and bacterial pathogens.

This idea that shorter protein isoforms fine-tune signalling is a common mechanism that occurs throughout the TLR signalling pathway. In response to LPS, myeloid differentiation primary response protein 88 (MYD88) enables the formation of multi-protein complexes that contain TLR4, MYD88, IL-1 receptor-associated kinase 1 (IRAK1) and IRAK4. IRAK1 is phosphorylated by IRAK4; phosphorylated IRAK1 binds to TNF receptorassociated factor 6 (TRAF6), and eventually NF- $\kappa$ B and activator protein 1 (AP-1) transcription factors are activated by IкB kinase (IKK) complexes (FIG. 2b). Stimulation of mouse monocytes with LPS or pro-inflammatory cytokines induces the expression of a splice variant of MYD88 - known as MYD88s - that lacks exon 2, which causes an in-frame deletion of the MYD88 intermediate domain ${ }^{21-23}$. Although MYD88s can still bind to TLRs and IRAK1, it cannot interact with IRAK4 (REF. 22). Consequently, MYD88s is unable to mediate IRAK1 phosphorylation and NF- $\kappa$ B activation ${ }^{21}$. MYD88s also acts as a dominant-negative inhibitor of NF- $\kappa \mathrm{B}$ signalling by forming heterodimers with full-length MYD88 (REF. 21). By contrast, MYD88s does not impair LPSinduced AP- 1 activation ${ }^{23}$. Thus, MYD88s production allows monocytes to differentially tune the NF- $\mathrm{BB}$ and AP-1 activation pathways.

Adding further complexity, IRAK1 is also subject to alternative splicing ${ }^{24,25}$. The IRAK $1 b^{24}$ and IRAK1-S 25 variants result from the use of alternative $3^{\prime}$ splice sites in exon 12. Both proteins lack kinase activity ${ }^{24,25}$ and IRAK1-S fails to bind TRAF6 (REF. 25). Nonetheless, both isoforms can induce NF- $\kappa$ B activation, possibly by forming functional heterodimers with full-length IRAK1 (REFS 24,25). Conversely, a third alternatively spliced variant that lacks exon 11, IRAK1c, has no kinase activity and acts as a dominant-negative inhibitor ${ }^{26}$. IRAK1c suppresses both NF- $\kappa \mathrm{B}$ activation and TNF production in response to LPS $^{26}$. IRAK2, another IRAK-like molecule, has four known alternatively spliced isoform $\mathrm{s}^{27}$. IRAK2a and IRAK2b potentiate NF- $\kappa$ B activation, whereas IRAK2c and IRAK2d act as inhibitors ${ }^{27}$. Finally (as reviewed in REF. 28), the NF- $\kappa B$ signalling cascade is tightly regulated by the expression of agonistic and antagonistic splice variants of inhibitor of NF- $\kappa B$ (IкB), IKK and the NF- $\kappa \mathrm{B}$ transcription factor subunits RELA (also known as the p65 subunit), RELB and NF- $\kappa B 2$ (also known as the $\mathrm{p} 100$ subunit).

Regarding the MYD88-independent TLR pathway, TLR3 stimulation induces the association of the adaptor molecule TIR-domain-containing adaptor protein inducing IFN $\beta$ (TRIF) with TRIS, which is a shorter splice variant of TRIF that lacks the Toll/IL-1R (TIR) domain ${ }^{29}$. Overexpression of TRIS activates NF- $\kappa \mathrm{B}$ and IFN-regulatory factor 3 (IRF3), whereas TRIS knockdown inhibits TLR3-mediated signalling ${ }^{29}$. These results suggest that the TLR3 signalling pathway involves the formation of heterocomplexes between TRIF and TRIS. TRIF-dependent TLR signalling also involves TRIF-related adaptor molecule (TRAM; also known as TICAM2) (FIG. 2b). In unstimulated cells, TRAM localizes to the plasma membrane where it interacts with TLR4 (REF. 30). In human mononuclear cells, a longer isoform of TRAM, known as TAG, results from the use of an alternative $3^{\prime}$ splice site in exon 4 of TRAM, and this variant contains an additional Golgi dynamics domain. Consequently, TAG localizes to the endoplasmic reticu$\operatorname{lum}(\mathrm{ER})^{30}$. Following stimulation with LPS, TRAM and TAG colocalize to late endosomes where TAG displaces the adaptor TRIF from its productive association with TRAM. TAG expression also promotes TRAM degradation. As a result, TAG inhibits LPS-induced IRF3 activation $^{30}$. Finally, IRF3 is also alternatively spliced, with eight different transcript variants described to date: IRF3, IRF3a to IRF3f, and IRF3CL ${ }^{31-33}$. Among them, only IRF3e is able to undergo cytoplasm-to-nuclear translocation in response to TLR3 ligands and bind to the IFNB promoter as full-length IRF3 does ${ }^{32}$. The other isoforms inhibit the transactivation potential of IRF3 to various degrees ${ }^{31-33}$.

Together, these studies reveal how alternative splicing and alternative polyadenylation are exceedingly common events that occur throughout innate immunity and fine-tune almost all steps in the process (FIG. 2b). Nevertheless, surprisingly little is known about the mechanisms that drive this alternative processing. What is known is that bacterial challenge of human DCs changes the mRNA levels of $>70$ splicing factors ${ }^{34}$ and LPS stimulation of mouse macrophages increases the mRNA and protein levels of CSTF64 (also known as CSTF2), which can favour the use of weak proximal polyadenylation sites ${ }^{34}$. Finally, two recent reports ${ }^{35,36}$ indicate that the kinetics of pre-mRNA splicing itself might regulate gene expression during innate immune responses. Transcriptome-wide analysis of lipid Astimulated macrophages revealed an accumulation of fully transcribed, but incompletely spliced, pre-mRNAs following TLR4 activation ${ }^{35}$. Similarly, TNF-induced splicing of intermediate and late transcripts is delayed compared with splicing of early gene pre-mRNAs ${ }^{36}$. These results suggest that not only are innate immune responses regulated by alternative pre-mRNA processing but the rate of such processing is also subject to variation, possibly to regulate the temporal order of gene expression in response to pro-inflammatory signals.

\section{mRNA stability in innate immunity}

Cellular mRNA levels are established by both mRNA production and degradation. Recently, in vivo labelling of newly synthesized RNAs using modified uridine (4-thiouridine $(4 \mathrm{sU})^{37}$ or bromodeoxyuridine $\left.(\mathrm{BrU})^{38}\right)$, or purification of chromatin-associated mRNAs ${ }^{35}$ enabled the simultaneous assessment of total and 
nascent transcript levels in cells stimulated with LPS ${ }^{35,37}$ or $\mathrm{TNF}^{38}$. As a result, both gene transcription and RNA decay rates could be evaluated for their respective contributions to cell responses. These analyses showed that increases in RNA levels that are induced by proinflammatory stimuli are mainly due to changes in the rate of transcription ${ }^{35,37}$. However, the duration of these responses - particularly those that are rapid and transient - is mainly determined by the rate of RNA decay ${ }^{37}$. In LPS-stimulated and TNF-stimulated macrophages, a negative correlation can be observed between the speed of transcript induction and intrinsic mRNA stability ${ }^{39,40}$. In addition, challenge with LPS $^{37}$, TNF $^{38}$ and Mycobacterium tuberculosis ${ }^{17}$ modulates the stability of numerous transcripts. For example, stimulation of fibroblasts with TNF induces stabilization of 152 mRNAs and destabilization of 58 other transcripts ${ }^{38}$. Similarly, LPS treatment of DCs alters the stability of $6 \%$ of the expressed mRNAs ${ }^{37}$. Interestingly, the affected transcripts are enriched for inflammatory and immune signalling genes, as well as NF- $\kappa \mathrm{B}$ targets ${ }^{37}$. Together, these results indicate that regulation of $\mathrm{mRNA}$ degradation is also essential for shaping innate immune responses.

ARE-mediated regulation of $m R N A$ stability. In 1986, conserved AU-rich sequences were discovered in the 3' UTR of the genes that encode the short-lived cytokines $\mathrm{TNF}^{41}$ and granulocyte-macrophage colony-stimulating factor (GM-CSF; which is encoded by CSF2 ${ }^{42}$. Insertion of the CSF2 AU-rich sequence into the 3' UTR of the stable transcript encoding $\beta$-globin was shown to strongly induce its degradation ${ }^{42}$. These studies pioneered the discovery of AREs as major regulators of mRNA stability. Approximately 5-8\% of all human transcripts contain $\mathrm{AREs}^{43,44}$ and many of these ARE-containing mRNAs are involved in inflammation ${ }^{43}$. Consistent with rapid mRNA decay being essential for controlling response duration, early and transient transcripts that are induced in LPS-stimulated or TNF-stimulated macrophages contain significantly more AREs in their $3^{\prime}$ UTRs than intermediate and late transcripts ${ }^{40}$. Moreover, numerous pro-inflammatory factors, as well as anti-inflammatory cytokines, undergo ARE-mediated regulation, including IL-6, IL-8, TNF, IL-1 $\beta$, GM-CSF, inducible nitric oxide synthase (iNOS; also known as NOS2), transforming growth factor- $\beta$ (TGF $\beta$ ) and IL-10 (REFS 45,46).

AREs consist of various large clusters of overlapping AUUUA pentamers and UUAUUUAUU nonamers that are specifically recognized by over 20 different ARE-binding proteins. Among them, tristetraprolin (TTP), butyrate response factor 1 (BRF1; also known as ZFP36L1), BRF2 (also known as ZFP36L2), KH-type splicing regulatory protein (KSRP; also known as KHSRP) and AU-rich element RNA-binding protein 1 (AUF1; also known as HNRNPD) stimulate target transcript decay by recruiting deadenylases and downstream degradation machineries ${ }^{45,46}$. By contrast, Y-box binding protein 1 (YB1; also known as NSEP1) and the ELAV (embryonic lethal and abnormal vision) family members Hu-antigen $\mathrm{R}$ (HUR; also known as ELAVL1) and HUD (also known as ELAVL4) stabilize their targets by competing with the destabilizing ARE-binding proteins for ARE occupancy $^{45,46}$ (FIG. 3a). ARE-mediated regulation of Tnf and Illb mRNA stability has been well studied. Notably, HUR initially stabilizes both transcripts in response to LPS ${ }^{47}$. LPS also induces TTP synthesis and phosphorylation ${ }^{48,49}$, and phosphorylated TTP is sequestered by the chaperone protein 14-3-3 (REF. 49). When dephosphorylated by protein phosphatase $2 \mathrm{~A}^{50}$, TTP displaces HUR, binding the Tnf ARE with high affinity and the Illb ARE with a lower affinity. TTP then recruits degradation factors to the Tnf transcript, but not to $I l 1 b^{48}$. The destabilizing protein AUF1 also targets Tnf and Il $1 b \mathrm{mRNAs}^{51}$. This regulation results in a rapid and transient induction of Tnf mRNA expression in response to LPS, whereas $I l 1 b$ mRNA is induced more slowly and has a longer half-life ${ }^{48}$. Mice that are deficient in TTP ${ }^{52,53}$ or AUF1 (REFS 51,54), or that express a mutant version of TNF that lacks its $\mathrm{ARE}^{47}$, develop severe inflammatory diseases ${ }^{52,53}$, including LPSinduced shock ${ }^{51,54}$. These symptoms, which result from excessive TNF and IL- $1 \beta$ production, illustrate the crucial role of ARE-mediated mRNA degradation in controlling inflammatory responses. Unexpectedly, mice that lack HUR expression in myeloid cells also show pathological exacerbation of their immune response $\mathrm{e}^{55}$. This outcome might result from HUR-mediated stabilization of antiinflammatory transcripts and/or inhibition of HURmediated translation in wild-type mice (see below). Together, these data highlight both the importance and the complexities of ARE-mediated post-transcriptional control of inflammation.

Non-ARE-mediated regulation of mRNA stability. The modulation of pro-inflammatory transcript stability also involves non-ARE regulatory elements. For example, a constitutive decay element (CDE) in the TNF 3' UTR confers an intrinsic short half-life to the transcript that is independent of ARE-mediated decay ${ }^{56}$. Recognition of embryo deadenylation element (EDEN)-like sequences - which are rich in uridine-purine dinucleotides - by CUG triplet repeat RNA-binding protein 1 (CUGBP1; also known as CELF1) additionally induces TNF and FOS mRNA deadenylation ${ }^{57}$. By contrast, polypyrimidine tract-binding protein (PTB; also known as PTBP1), which is induced by pro-inflammatory cytokines, stabilizes iNOS transcripts through the recognition of a UC-rich sequence in the $3^{\prime} \mathrm{UTR}^{58}$ (FIG. 3b).

Among 3' UTR regulatory elements, miRNAs have emerged as key modulators of mRNA decay and translation. They consist of $~ 21$-nucleotide-long non-coding RNAs that base-pair to partially complementary sequences in the 3' UTR of their target RNAs. miRNAs act as the nucleic acid core of the RNA-induced silencing complex (RISC), which inhibits mRNA translation and/or causes deadenylation and the subsequent decay of target transcripts ${ }^{59}$ (FIG. 3C). More than 1,000 miRNAs have been identified in the human genome ${ }^{60}$ and as many as $60 \%$ of all mRNAs are predicted to contain a miRNA target site (or multiple sites) ${ }^{61}$. Abundant evidence has revealed the importance of miRNAs in the development of immune cells, as well as in the initiation and termination of inflammation (reviewed in REFS 62,63). 


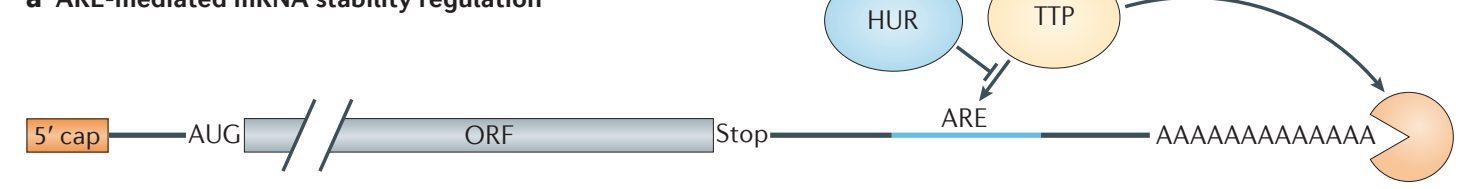

b Non-ARE-mediated mRNA stability regulation

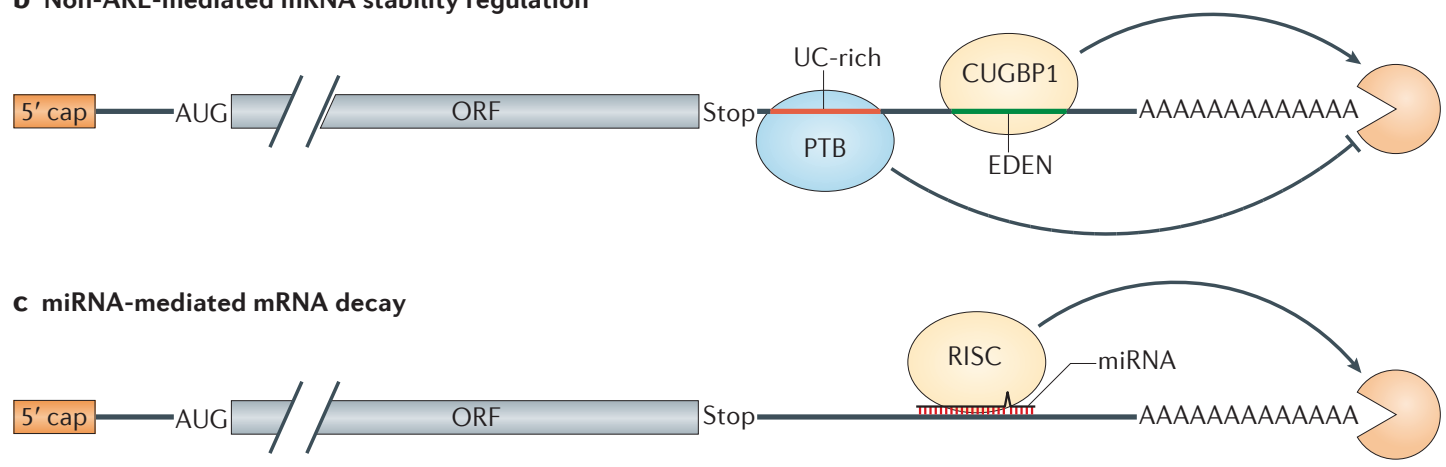

d Nonsense-mediated mRNA decay

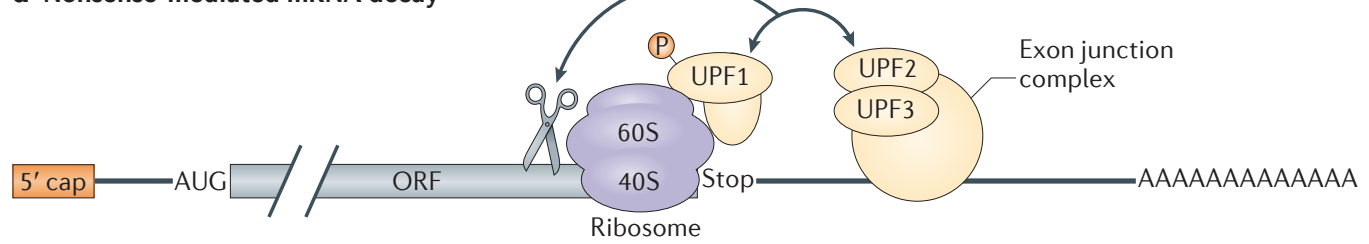

Figure 3 | Regulation of mRNA stability during innate immune responses. a | Many cytokine transcripts contain AU-rich elements (AREs) in their 3' untranslated regions (3' UTRs). The recognition of these motifs by destabilizing ARE-binding proteins, such as tristetraprolin (TTP), stimulates mRNA deadenylation and decay. Conversely, the binding of stabilizing proteins - such as Hu-antigen R (HUR) — that compete with destabilizing factors inhibits ARE-mediated RNA degradation. $\mathbf{b}$ | The recognition of other regulatory elements, such as embryo deadenylation element (EDEN)-like sequences by CUG triplet repeat RNA-binding protein 1 (CUGBP1) can additionally stimulate RNA deadenylation, whereas binding of polypyrimidine tract-binding protein (PTB) to UC-rich sequences stabilizes mRNAs. c|Numerous transcripts that are involved in innate immune responses also contain a microRNA (miRNA) target site (or multiple sites) in their 3' UTRs. Specific recognition of these sites by the RNA-induced silencing complex (RISC) leads to deadenylation of the mRNA and its subsequent degradation. $\mathbf{d}$ | Finally, the presence of an exon junction complex downstream of a stop codon of a translated mRNA induces nonsense-mediated decay through interactions between regulator of nonsense transcripts (UPF) proteins, phosphorylation of UPF1 and endonucleolytic cleavage of the transcript. ORF, open reading frame.

Finally, transcripts that contain a very long 3' UTR or an exon junction complex downstream of the translation termination codon can be degraded by nonsensemediated decay (NMD) (FIG. 3d). This mechanism prevents the production of deleterious truncated proteins that are encoded by mutant or aberrantly spliced mRNAs containing premature termination codons. However, accumulating evidence shows that there is conserved expression of transcripts that are naturally spliced in their $3^{\prime} \mathrm{UTR}^{64}$, notably in haematopoietic cells. Inhibition of NMD impairs haematopoiesis ${ }^{65}$ and deletion of the NMD factor regulator of nonsense transcripts 2 (UPF2) induces the upregulation of 186 genes in macrophages ${ }^{65}$. These results suggest that, in addition to its function as a quality control mechanism, NMD regulates gene expression in innate immune cells by controlling transcript stability.

\section{Translation initiation in innate immunity}

Many signalling events in innate immunity require gene expression changes that are too fast for new transcription or alternative pre-mRNA processing. In this case, changes in the translation of pre-existing mRNAs can allow for more rapid dynamic responses. Illustrating the importance of this post-transcriptional regulatory mechanism, LPS stimulation of DCs induces an immediate and massive increase in new protein synthesis within the first 60 minutes $^{66}$.

Regulation of translation initiation factor activity. Among all translation initiation factors, eukaryotic translation initiation factor 2 (eIF2) is the best studied regulator in innate immunity. eIF2 forms a ternary complex with the initiator methionyl-tRNA and a molecule of GTP, and this complex binds to the 40S ribosomal 
subunit where it is essential for start codon recognition and recruitment of the $60 \mathrm{~S}$ ribosomal subunit. Upon positioning of the $40 \mathrm{~S}$ subunit at the start codon, eIF2 hydrolyses its bound GTP, which causes the release of eIF2 from the ribosome (FIG. 4a). The resulting eIF2-GDP is then recycled by the guanine nucleotide exchange factor eIF2B to form a new ternary complex that is competent for a new round of translation. The activity of eIF2 is regulated by four different kinases that phosphorylate its $\alpha$-subunit (eIF2 $\alpha$ ) and block its recycling by eIF2B. The phosphorylation of eIF2 can be triggered by double-stranded RNA (through protein kinase RNA-activated (PKR; also known as eIF2AK2)), ER stress (through PKR-like ER kinase (PERK; also known as eIF2AK3)), exposure to ultraviolet light (through GCN2; also known as eIF2AK4) or haem deficiency (through haem-regulated inhibitor (HRI; also known as eIF2AK1)). The phosphorylation of eIF2 leads to global translational repression of most cellular and viral mRNAs ${ }^{67}$. Suppression of translation mediated by eIF2 phosphorylation is beneficial during viral infection as it blocks the production of new viral proteins and limits viral spread. However, under the pathological chronic ER stress, prolonged eIF2 phosphorylation can be deleterious and lead to apoptosis ${ }^{68}$. Interestingly, TLR 3 or TLR4 activation in macrophages and fibroblasts leads to the dephosphorylation of eIF2B via TRIF $^{69,70}$. As a consequence, the guanine exchange activity of eIF2B is strongly stimulated and recycling of eIF2 occurs even though eIF2 $\alpha$ remains phosphorylated (FIG. 4a). This allows the maintenance of efficient mRNA translation rates and an increase in cell survival upon prolonged ER stress, while still benefitting from the unfolded protein response (UPR) that is triggered by the ER stress and is essential to restore protein-folding homeostasis in the cell.

In addition to eIF2, the cap-binding protein eIF4E is highly regulated. eIF4E mediates the recruitment of the $40 \mathrm{~S}$ ribosomal subunit by interacting both with the $5 '$ mRNA cap structure and the scaffold initiation factor eIF4G, which in turn contacts the $40 \mathrm{~S}$ ribosome through eIF3 (FIG. 4b). In most cells, eIF4E levels are limiting, and thus the regulation of its activity has a strong impact on the translation efficiency of many mRNAs. Notably, eIF4E phosphorylation was recently shown to regulate the translation of pro-tumorigenic mRNAs ${ }^{71}$, and eIF4E phosphorylation is usually altered in response to viral infection, which suggests a potential role in regulating innate immunity ${ }^{72}$. Consistent with this, mice that lack the two MAPK-interacting protein kinases (MNK1 and MNK2) that are responsible for eIF4E phosphorylation (FIG. 4b), or that express a mutant form of eIF4E that cannot be phosphorylated, have an enhanced type I IFN response that blocks infection by RNA viruses ${ }^{73}$. Surprisingly, although the lack of eIF4E phosphorylation does not affect global mRNA translation, it leads to specific translational downregulation of many mRNAs, including the mRNA that encodes I $\kappa \mathrm{B} a$. This increases $N F-\kappa B$ expression following RNA virus infection or specific TLR3 activation, which results in the induction of mRNAs that encode IFN $\beta$ and IRF7.
The phosphorylation of eIF4E is also regulated by IRAK2 and IRAKM (also known as IRAK3) (FIG. 4b). It has been shown that MNK1 and eIF4E were hypophosphorylated upon LPS stimulation in IRAK2deficient mice compared with wild-type mice ${ }^{74}$. Consistent with low eIF4E phosphorylation levels, translation of several cytokines (including TNF and IL-6) was less efficient in IRAK2-deficient macrophages in response to LPS stimulation. Thus, in addition to its role in promoting NF- $\kappa \mathrm{B}$ induction, IRAK2 promotes the translation of pro-inflammatory cytokines. Interestingly, IRAKM was recently shown to interact with IRAK2 and inhibit its ability to phosphorylate eIF4E (FIG. 4b), thereby preventing increased translation of cytokine mRNAs $s^{75}$. This inhibitory effect is thought to be important for downregulating TLR responses.

The activity of translation initiation factors is also subject to regulation by lipid mediators. In alveolar macrophages that are exposed to prolonged LPS treatment, 15-deoxy- $\Delta$-12,14-prostaglandin J2 (15d-PGJ2) - a prostaglandin with anti-inflammatory activity inhibits eIF4A activity and induces the formation of stress granules $^{76}$. eIF4A is a DEAD-box RNA helicase that is required to unwind any RNA secondary structures that might otherwise block $40 \mathrm{~S}$ ribosome progression through the $5^{\prime}$ UTR to find the start codon. Impairment of eIF4A activity by 15d-PGJ2 leads to translational repression of most cellular mRNAs, as well as sequestration of the pro-inflammatory TRAF2 protein into stress granules to resolve chronic inflammatory responses ${ }^{76}$.

Together, these studies illustrate the diversity of mechanisms by which translation initiation factor activity is controlled by phosphorylation or direct interaction with small molecules to modulate both activation and resolution of inflammation.

Regulation by mTOR and 4EBPs. Mammalian target of rapamycin (mTOR) is a serine/threonine kinase that responds to many cellular stimuli, including TLR ligands. Its activation in macrophages occurs through MYD88-TRIF-phosphoinositide 3-kinase (PI3K)-AKT pathways ${ }^{77}$. In addition to regulating the transcription of immune genes, mTOR mediates the phosphorylation of eIF4E-binding proteins (4EBPs) (FIG. 4b). When hypophosphorylated, 4EBPs bind and sequester the translation initiation factor eIF4E to block its association with the scaffold initiation factor eIF4G and repress cap-dependent translation. Upon mTOR activation, 4EBPs become hyperphosphorylated and release eIF4E, which is then available to bind to eIF4G and participate in translation (FIG. 4b). The importance of $4 \mathrm{EBPs}$ in the translational control of innate immunity was revealed in mice that lack both $4 \mathrm{EBP} 1$ and $4 \mathrm{EBP} 2$ (Eif4ebp $1^{-/-}$Eif4ebp $2^{-/-}$mice), which are refractory to RNA virus infection ${ }^{78}$. Further analysis revealed that 4EBP-depleted cells have increased type I IFN production following exposure to polyinosinic:polycytidylic acid (poly(I:C)) or in response to viral infection. Interestingly, although eIF4E is required for the translation of most cellular mRNAs, its sequestration by $4 \mathrm{EBPs}$ mainly affects the expression of those transcripts with 
a Regulation of elF2 activity
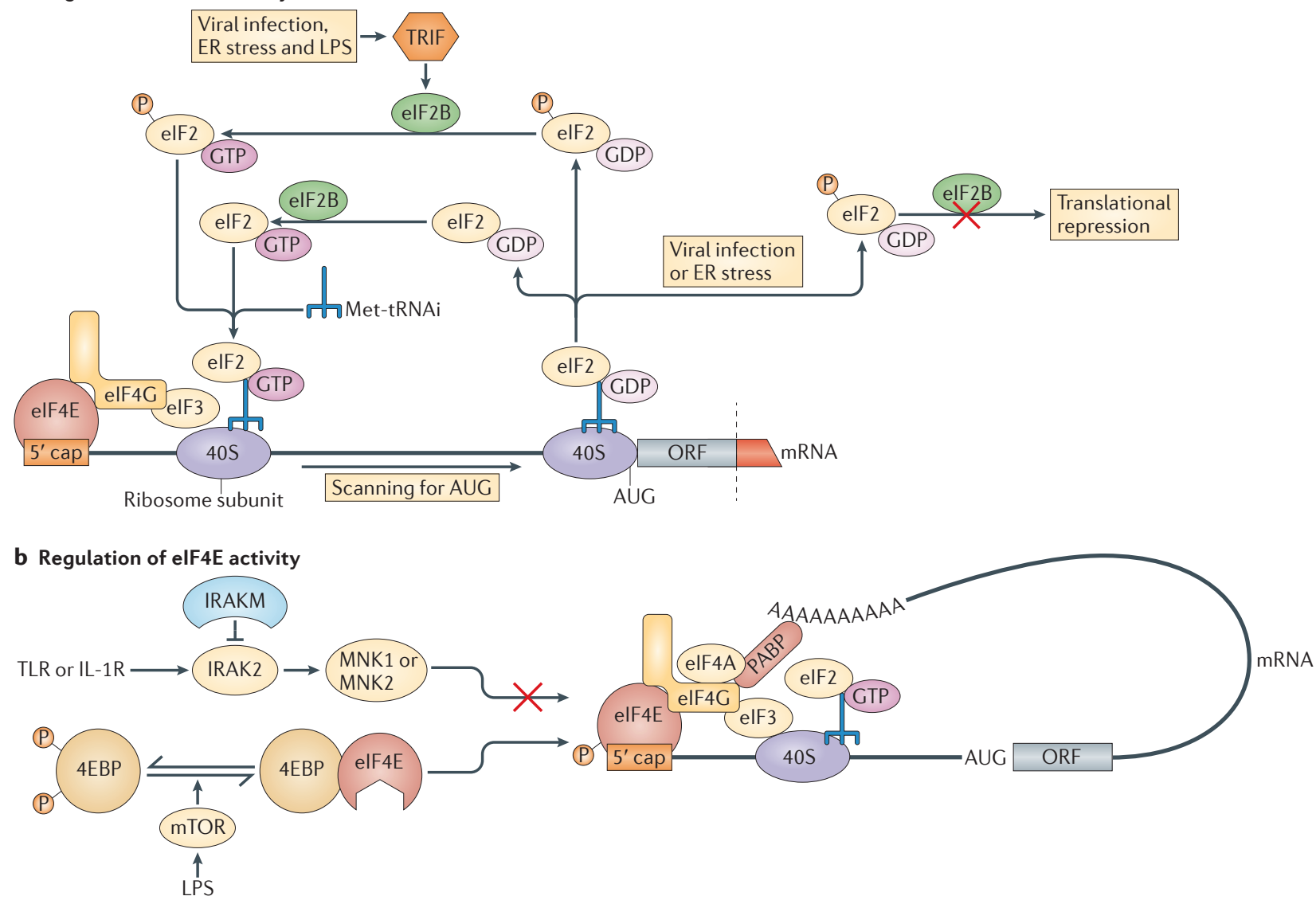

\section{c Translation re-initiation}

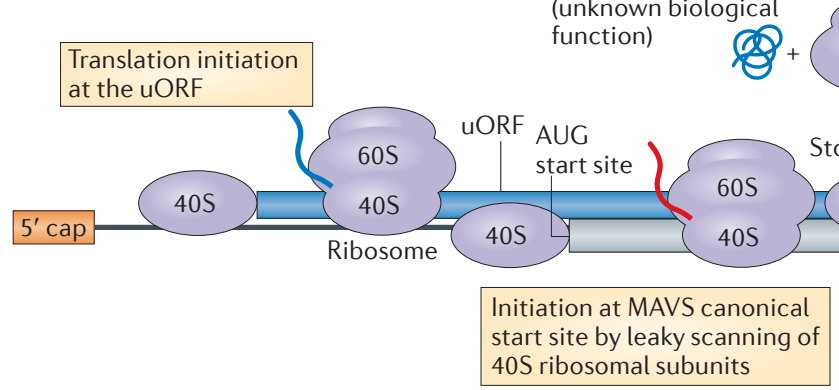

Figure 4 | Translation initiation control of innate immunity.

a | Regulation of eukaryotic translation initiation factor 2 (elF2) activity. Under normal conditions, elF2 associates with a GTP molecule, a methionine-initiator tRNA (Met-tRNAi) and the $40 \mathrm{~S}$ ribosome to participate in translation initiation. After initiation, the GTP molecule is hydrolysed and elF2 is released from the $40 \mathrm{~S}$ ribosome. The GDP-associated elF2 is then recycled by eIF2B into a GTP-associated eIF2 that can re-engage in translation. During viral infection or endoplasmic reticulum (ER) stress, elF2 can be phosphorylated, which impairs its recycling by elF2B, leading to translational inhibition of most mRNAs. Toll-like receptor (TLR) engagement under ER stress conditions leads to elF2B stimulation, which in turn is able to efficiently recycle elF2, even in its phosphorylated form, to maintain translation. $\mathbf{b}$ | Regulation of elF4E activity. TLR or interleukin-1 receptor (IL-1R) engagement induces the phosphorylation of elF4E in an IL-1R-associated kinase 2 (IRAK2)-dependent and MAPK-interacting protein kinase 1 (MNK1)-dependent or MNK2-dependent manner to stimulate the translation of a subset of mRNAs. TLR engagement also activates the mammalian target of rapamycin (mTOR) pathway, which leads to elF4E-binding protein (4EBP) phosphorylation, thus releasing the cap-binding protein elF4E to stimulate the translation of mRNAs with highly structured 5' untranslated regions (5' UTRs). c| Translation re-initiation. A large proportion of cellular transcripts have predicted short upstream open reading frames (uORFs). When translated, these uORFs can affect the expression of the canonical ORF by different means. If the uORF overlaps with the main ORF, its translation will downregulate the translation of the main ORF, which will depend exclusively on leaky scanning of 40 S ribosomal subunits that fail to recognize the start codon of the uORF and continue scanning the $5^{\prime}$ UTR until they reach the canonical ORF start codon - in this case the full-length mitochondrial antiviral signalling protein (FL-MAVS). Ribosomes that terminate translation of the uORF sometimes fail to dissociate from the mRNA, and the $40 \mathrm{~S}$ ribosomal subunit might re-initiate scanning in a $5^{\prime}$ to $3^{\prime}$ direction until the ribosomes reach a new start codon situated in an optimal Kozak context. In this case, if the start codon is in the same reading frame as that of the canonical ORF, translation re-initiation will produce a truncated version of the canonical protein that is synthesized from the canonical ORF (in this case, the truncated version is called miniMAVS). If the internal start codon is not in the same reading frame, it can lead to the synthesis of a completely different protein. IFN, interferon; LPS, lipopolysaccharide; PABP, poly(A)-binding protein; TRIF, TIR-domain-containing adaptor inducing IFN $\beta$. 
large secondary structures in their $5^{\prime}$ UTR and those that contain 5' UTR oligopyrimidine tracts. Both of these UTR classes are highly dependent on eIF4E for efficient translation ${ }^{79,80}$. Among these genes, translation of IRF7 - which has a long and highly structured $5^{\prime}$ UTR - is stimulated in cells in which 4EBP1 and $4 \mathrm{EBP} 2$ are depleted. Consistent with a role of $4 \mathrm{EBPs}$ in regulating innate immunity-related genes, LPS-mediated activation of macrophages leads to mTOR-dependent 4EBP phosphorylation, which activates the translation of TNF, IL-6 and CXC-chemokine ligand 1 (CXCL1) ${ }^{79}$. Thus, 4EBPs act as negative regulators of innate immunity in unstimulated cells and are required both for inducing efficient expression of IFN-regulatory genes as well as for avoiding an excessive innate immune response against pathogens. In agreement with such an important role, inactivation of mTOR by the Leishmania spp. protease GP63 (also known as leishmanolysin) leads to translational repression of macrophage transcripts and is required for pathogen survival ${ }^{81}$.

In contrast to these findings, mTOR inactivation by rapamycin during the course of a bacterial infection has been shown to stimulate innate immunity by favouring the expression of pro-inflammatory genes ${ }^{82}$. Furthermore, infection of macrophages with a virulent strain of Legionella pneumophilia results in mTOR ubiquitylation and degradation, thereby suppressing its function ${ }^{83}$. Surprisingly, in this case, the resulting hypophosphorylation of 4EBPs leads to translational repression of lowabundance transcripts and activation of high-abundance transcripts. Among these abundant transcripts are those for pro-inflammatory cytokines. Interestingly, mTOR inactivation by L. pneumophilia requires the Dot/lcm secretion system, which suggests that triggering the innate immune system involves translational regulation following the detection of pathogen signatures.

The above data demonstrate the importance of translational regulation mediated by mTOR and 4EBPs in innate immunity. These data further illustrate the dual role of $4 \mathrm{EBPs}$ in restricting or promoting innate immunity depending on the nature of the pathogen.

Regulation of poly (A) length. The poly(A) tail located at mRNA 3' ends has an essential role in translation by serving as a binding site for poly(A)-binding protein (PABP; also known as PABP1). Although recruited to the $3^{\prime}$ end, PABP interacts with multiple translation initiation factors and stimulates their activities (FIG. 4b). These interactions also bring the $5^{\prime}$ and $3^{\prime}$ ends into close proximity, thereby pseudo-circularizing the mRNA, which is thought to improve ribosome recycling and therefore translational efficiency ${ }^{84}$. Dynamic regulation of poly $(\mathrm{A})$ tail length in numerous cell types has a strong impact on both translational efficiency and transcript stability ${ }^{85}$.

In unstimulated macrophages, TNF mRNA is constitutively expressed but it lacks a poly(A) tail and so fails to engage the translation machinery and produce TNF protein ${ }^{86}$. However, following LPS stimulation, TNF transcripts gain poly(A) tails, which activates their translation and allows the rapid and abundant expression of TNF protein. Such regulation is similar to that occurring in resting memory $\mathrm{CD}^{+} \mathrm{T}$ cells, in which constitutively expressed mRNA that encodes CC-chemokine ligand 5 (CCL5) lacks a poly(A) tail and so is translationally repressed until the T cell receptor is re-engaged. This re-engagement triggers polyadenylation of the pre-existing pool of CCL5 mRNA, which facilitates rapid translation and CCL5 protein secretion ${ }^{87}$. Interestingly, although the mechanism responsible for the deadenylation and subsequent readenylation of TNF has not been elucidated, the AU-rich elements that are located within its 3' UTR are very similar in sequence to the motif that is recognized by the cytoplasmic polyadenylation element binding protein (CPEB; also known as CPEBP1). CPEB has been shown to regulate the translation of mRNAs for many pro-inflammatory cytokines (including IL-6) in mouse embryonic fibroblasts ${ }^{88}$. It is therefore possible that, in addition to TNF, many other transcripts may be constitutively produced in resting macrophages and stored in a translationally silent state until TLR engagement triggers their rapid readenylation and translation.

\section{Alternative translation initiation pathways}

Although most mRNAs are translated through the classical cap-dependent mechanism, a subset of cellular mRNAs can also rely on alternative ways to initiate translation, such as leaky scanning, non-AUG translation initiation, translation re-initiation and internal ribosome entry sites (IRESs).

Recognition of the start codon by the scanning $43 \mathrm{~S}$ ribosome is modulated by the nucleotide sequence surrounding the AUG, which is also known as the Kozak context ${ }^{89}$. The optimal sequence corresponds to a purine at position -3 and a guanosine at position +1 . If the Kozak context is not optimal, the $43 \mathrm{~S}$ ribosome fails to recognize the AUG codon and continues its $5^{\prime}$ to $3^{\prime}$ scanning until it reaches a downstream start codon - this mechanism is known as leaky scanning. Leaky scanning occurs in a variety of transcripts and allows the expression of multiple isoforms of the same protein without the requirement for alternative splicing. In DCs, translation of the transcript that encodes the secreted protein osteopontin (also known as SPP1) is controlled by leaky scanning to produce full-length secreted osteopontin and an amino-terminal truncated osteopontin isoform that is restricted to the cytoplasm ${ }^{90}$. Interestingly, translation of the $\mathrm{N}$-terminal isoform is not initiated at an AUG codon but probably at a GCC codon (coding for aspartic acid) that is located downstream of the canonical AUG. Expression of this N-terminal truncated osteopontin isoform is required for efficient podosome formation upon DC activation by $\mathrm{CpG}$-containing oligonucleotides ${ }^{90}$.

Translation re-initiation occurs when an 80 S ribosome that terminates translation at the stop codon is not completely recycled and the $40 \mathrm{~S}$ ribosomal subunit is able to resume $5^{\prime}$ to $3^{\prime}$ scanning to reach a downstream initiation codon and re-initiate translation. The efficiency of re-initiation is linked to the length of the first open reading frame $(\mathrm{ORF})$ that is translated, with shorter 
ORFs allowing for a more efficient re-initiation ${ }^{91}$. Indeed, it is thought that translation initiation factors (which are required for translation re-initiation) remain associated with ribosomal subunits for some time after elongation begins and, therefore, ribosomes that are translating short ORFs will have more chance of carrying all of the factors that are necessary for re-initiation. Interestingly, more than $45 \%$ of mammalian mRNAs are predicted to contain small upstream ORF (uORF) in their $5^{\prime} \mathrm{UTR}^{92}$, which suggests that they could have a widespread role in regulating translation of the main ORF. In a recent report, two isoforms of the antiviral retinoic acidinducible gene I (RIG-I) adaptor protein mitochondrial antiviral signalling protein (MAVS) - full length MAVS (FL-MAVS) and an N-terminal truncated isoform (miniMAVS) - were shown to be expressed from a single transcript species through the use of two in-frame start codons ${ }^{93}$. FL-MAVS is responsible for efficient type I IFN production during viral infection, whereas miniMAVS antagonizes FL-MAVS. Surprisingly, when dissecting the molecular mechanism responsible for miniMAVS translation, the authors revealed the presence of a short UORF in the $5^{\prime}$ UTR of the MAVS transcript that terminates downstream of the FL-MAVS start codon (FIG. 4c). Translation of this uORF allows ribosomes to bypass the FL-MAVS start codon. Then, through a mechanism of translation re-initiation, ribosomes can resume scanning and reach the start codon for translation of miniMAVS (FIG. 4C). By contrast, translation of FL-MAVS occurs through a leaky scanning mechanism whereby $40 \mathrm{~S}$ ribosomal subunits fail to recognize the uORF start codon and continue scanning until they reach the start codon for FL-MAVS (FIG. 4C). The ratio of FL-MAVS and miniMAVS is dynamic during the course of viral infection, which suggests that leaky scanning and translation re-initiation can be differentially regulated. Finally, by performing genomewide ribosome-footprinting experiments, numerous genes with multiple translation start sites have been identified, including genes that are involved in innate immunity, which demonstrates the widespread use of alternative translation initiation codons to increase the coding potential of mRNAs without involving alternative splicing.

In addition to translation re-initiation, some cellular transcripts rely on IRESs to initiate their translation. IRESs are RNA elements that can, through their secondary structure or primary sequence, recruit a $40 \mathrm{~S}$ ribosomal subunit independently of the mRNA $5^{\prime}$ cap structure and the cap-binding factor eIF4E (reviewed in REF. 84). Ribosome recruitment occurs through direct interactions between components of the translation machinery (including translation initiation factors) and the RNA sequence or structure, and can be regulated by IRES trans-acting factors. Although translation that is mediated by cellular IRESs is usually inefficient under normal conditions, it allows translation to be sustained during conditions where cap-dependent translation is compromised. A few genes that are involved in innate immunity have been reported to contain IRESs in their 5' UTR, including hypoxia-inducible factor $1 \alpha(\mathrm{HIF} 1 \alpha)$ and human surfactant protein A (SPA; also known as PSPA) ${ }^{94,95}$. However, IRES activity for these transcripts has not yet been monitored in the context of innate immunity. By contrast, polysome profiling of breast cancer cells that had been incubated with conditioned medium from activated macrophages revealed the genes for which translation was upregulated in the context of an inflammatory response ${ }^{96}$. Among these genes, early growth response gene 2 (EGR2) and 1,25-dihydroxyvitamin D3 24-hydroxylase (CYP24A1) were reported to depend on IRESs for their translation under inflammatory conditions ${ }^{96,97}$. As innate immunity is often associated with cellular stress conditions in which cap-dependent translation is highly regulated, it is tempting to speculate that IRES-mediated translation could have a role in allowing the translation of transcripts that are required to cope with such stresses.

\section{Gene-specific regulation}

Translation can be regulated in a transcript-specific manner through the recruitment of RNA-binding proteins, lncRNAs or small RNAs (FIG. 5a,b). Such interactions can occur on the $5^{\prime}$ UTR, the coding sequence or the $3^{\prime}$ UTR of target mRNAs and depend either on the transcript primary sequence or on particular RNA secondary structures.

Regulation by ARE-binding proteins. ARE-binding proteins are among the most important TLR-dependent regulators of translation. In addition to their role in modulating mRNA stability (see above), ARE-binding proteins have been reported to regulate the translation of key ARE-containing mRNAs following TLR engagement. Interestingly, because different ARE-binding proteins recognize similar sequence motifs, they can compete with one another for individual AREs and simultaneously occupy a single transcript that contains multiple AREs ${ }^{98}$ (FIG. 5a). This results in complex and dynamic regulatory networks, which possibly involve multiple molecular mechanisms that affect both transcript translation and stability. Illustrating this, translation of TNF in resting macrophages is repressed by the ARE-binding protein TTP. However, following LPS stimulation, activation of the p38 mitogenactivated protein kinase (MAPK)-MAPK-activated protein kinase 2 (MAPKAPK2) pathway leads to TTP phosphorylation, which decreases its affinity for TNF AREs. As a consequence, TTP is replaced by HUR, which stimulates TNF translation ${ }^{99}$.

The exact molecular mechanisms by which AREbinding proteins regulate translation remain largely unexplored but most probably depend on the recruitment of additional proteins. In resting macrophages, TTP was shown to interact with DEAD-box protein 6 (DDX6; also known as RCK) and repress TNF translation, possibly by recruiting the mRNA to processing bodies (P-bodies) ${ }^{100}$. Nucleolysin TIA1 isoform p40 (TIA1), an ARE-binding protein that is required for translational regulation of TNF and other cytokines following TLR activation, has been shown to repress 


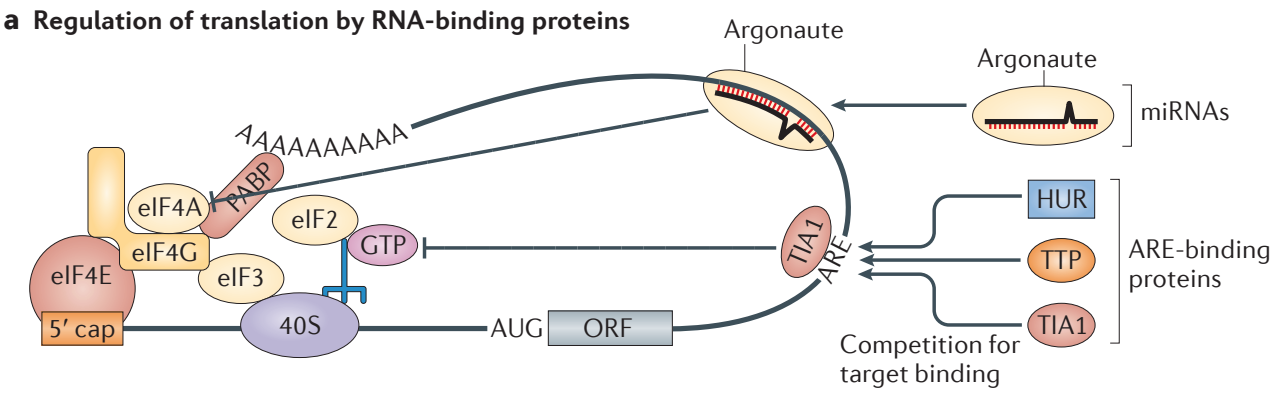

\section{b Regulation of translation by the GAIT complex}

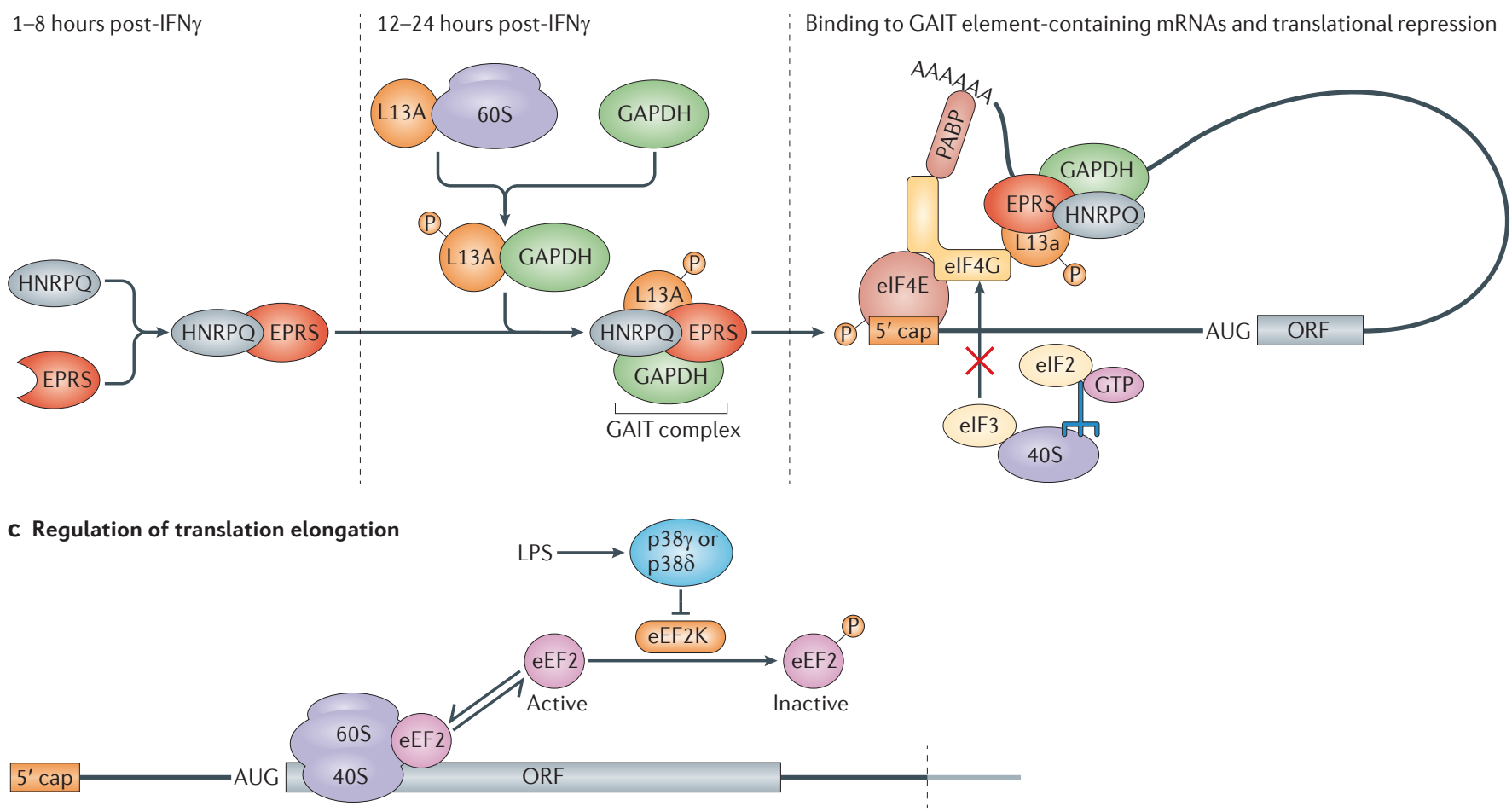

Figure 5 | Translational control mediated by RNA-binding proteins and elongation factors. a|Translational control through RNA-binding proteins. Differential expression of microRNAs (miRNAs) during Toll-like receptor (TLR) signalling can lead to translational repression of immune-related mRNAs. This inhibition is thought to occur mainly at the level of translation initiation through targeting of scanning by the $40 \mathrm{~S}$ ribosomal subunit ${ }^{125}$. miRNAs can also lead to target transcript deadenylation to block translation initiation. TLR signalling regulates the levels and activity of many AU-rich element (ARE)-binding proteins, which are thought to regulate translation initiation through mechanisms that are still not fully elucidated. $\mathbf{b} \mid$ Interferon- $\gamma$ (IFN $\gamma$ ) induces the multistep assembly of an IFN $\gamma$-activated inhibitor of translation (GAIT) complex through the association of glutamyl-prolyl tRNA synthetase (EPRS) and heterogeneous nuclear ribonucleoprotein Q (HNRPQ) 8 hours after IFN $\gamma$ treatment, which is followed by the association of the large ribosomal subunit protein L13A with glyceraldehyde 3-phosphate dehydrogenase (GAPDH) and the formation of the fully functional GAIT complex. The GAIT complex binds to the 3' untranslated region (UTR) of transcripts containing the GAIT element and represses their translation by abolishing the interaction between eukaryotic translation initiation factor 4G1 (elF4G) and elF3.c|Regulation of translation elongation. In macrophages, lipopolysaccharide (LPS) stimulation inhibits, in a mitogenactivated protein kinase (MAPK)-dependent manner, the kinase activity of eukaryotic elongation factor 2 kinase (eEF2K), thus increasing the pool of active eEF2 in the cell and stimulating translation elongation. HUR, Hu-antigen R; ORF, open reading frame; PABP, poly(A)-binding protein; TIA1, nucleolysin TIA1 isoform p40; TTP, tristetraprolin.

the translation of target mRNAs by preventing their engagement with polyribosomes ${ }^{101}$. Although the mechanism of this TIA1-dependent translational repression has not been fully elucidated, it has been suggested that TIA1 promotes the assembly of $48 \mathrm{~S}$-like ribosomes that lack eIF2 and are therefore unable to initiate translation ${ }^{102}$. This would be consistent with the known role of TIA1 in repressing the translation of mRNAs with 5' UTR oligopyrimidine tracts under stress conditions - when eIF2 $\alpha$ is phosphorylated and thus unavailable for translation - by relocalizing these mRNAs to stress granules ${ }^{103}$. 
Regulation by the GAIT complex. In addition to AREbinding proteins, the IFN $\gamma$-activated inhibitor of translation (GAIT) complex has an important role in gene-specific translational control in innate immunity. Evidence for the GAIT complex was first found in IFN $\gamma$ treated human monocytic U937 cells in which translation of the mRNA encoding ceruloplasmin (CP) was first stimulated and then strongly repressed after 16 hours of treatment ${ }^{104}$. Later, a 29 -nucleotide bipartite stem-loop RNA structure that is located in the $3^{\prime}$ UTR of the $C P$ transcript was reported to interact with a protein complex and shown to be sufficient to mediate translational repression of $C P$ and that of reporter constructs expressed in IFN $\gamma$-treated cells ${ }^{105}$. Identification of the protein partners involved in GAIT - carried out using a yeast threehybrid screen and affinity chromatography - revealed a $450 \mathrm{kDa}$ complex that is composed of the large ribosomal subunit protein L13A (also known as RPL13A), glutamylprolyl tRNA synthetase (also known as EPRS and bifunctional glutamate/proline tRNA ligase), heterogeneous nuclear ribonucleoprotein Q (HNRPQ; also known as NSAP1) and glyceraldehyde-3-phosphate dehydrogenase $(\mathrm{GAPDH})^{106,107}$. Interestingly, the GAIT complex is assembled in a two-step process in which, 8 hours after IFN $\gamma$ treatment, EPRS and HNRPQ first assemble together but are unable to bind to GAIT elementcontaining mRNAs ${ }^{106}$ (FIG. 5b). After 12 to 24 hours of treatment, L13A is phosphorylated and released from the $60 \mathrm{~S}$ ribosomal subunit, which allows its interaction with GAPDH and with the EPRS-HNRPQ heterodimer ${ }^{106,107}$ (FIG. 5b). The formed complex can then interact with the GAIT RNA element and drive translational repression by a mechanism that involves the direct interaction of $\mathrm{L} 13 \mathrm{~A}$ with the translation initiation factor eIF $4 \mathrm{G}^{108}$. The L13A-eIF4G interaction interferes with the association of eIF4G with eIF3 and thus blocks the recruitment of the $40 \mathrm{~S}$ ribosomal subunit to the target $\mathrm{mRNA}^{108}$ (FIG. 5b).

In addition to regulating translation of the $C P$ transcript, a polysome-profiling experiment combined with microarray analysis of IFN $\gamma$-treated cells revealed that many other mRNAs are also regulated by the GAIT complex, including chemokines and chemokine receptors $^{109}$, as well as genes that are involved in regulating GAIT complex assembly ${ }^{110}$. Among these genes, vascular endothelial growth factor A (VEGFA), which has a role in promoting angiogenesis during inflammation, was shown to contain a GAIT element in its 3' UTR that was able to recruit the GAIT complex and repress VEGFA translation ${ }^{111}$. The GAIT RNA element that is located in the 3' UTR of VEGFA is in close proximity to a binding site for the RNA-binding heterogeneous nuclear ribonucleoprotein L (HNRNPL) in complex with the double-stranded RNA-binding protein DRBP76 (also known as ILF3) and HNRNPA2/B1; this is also known as the HILDA complex. Binding of the GAIT complex and HNRNPL is mutually exclusive and mediated by a differential conformational change of the target RNA induced by each complex that, in turn, blocks the binding of the other complex ${ }^{112,113}$. This conformational switch allows the fine-tuning of VEGFA translation in the course of inflammation. Under normoxic conditions, IFN $\gamma$ treatment activates the GAIT complex, which binds to the 3' UTR of VEGFA to inhibit its translation. However, during hypoxia, HNRNPL is phosphorylated and relocalizes to the cytoplasm and binds to the 3' UTR of $V E G F A$, thus impeding GAIT complex binding to allow for efficient VEGFA protein expression and to promote angiogenesis ${ }^{113}$.

Together, available data exemplify the complexity and dynamic aspect of gene-specific translational control in innate immunity. Indeed, simultaneous binding and competition for binding sites between different RNA-binding proteins allows the cell to integrate multiple inputs at the same time and to differentially regulate gene expression in a target-specific manner as appropriate. Furthermore, it introduces the notion of a post-transcriptional code for regulating gene expression whereby the combinatorial binding of RNA-binding proteins to a particular transcript determines its expression level.

\section{Regulation of translation elongation}

Although most regulation of translation is thought to occur at the initiation step, translation can also be controlled at the elongation step. However, the mechanisms for regulating elongation, as well as their impact in physiological processes, are still poorly understood. It is known that translation elongation can be regulated by the mTOR and MAPK pathways in response to many stimuli ${ }^{79}$. Among these, TLR activation in macrophages that are deficient in MAPK kinase kinase 8 (MAP3K8; also known as COT and TPL2) results in reduced phosphorylation of eukaryotic elongation factor 2 kinase (eEF2K), which suggests a role for MAP3K8 in the regulation of translation elongation ${ }^{79}$. In its unphosphorylated form, eEF2K acts as a translational repressor by phosphorylating eEF2 (FIG. 5c). Confirming an involvement of eEF2 in innate immunity, activation of the MAPK proteins $\mathrm{p} 38 \gamma$ (also known as MAPK12) and p38 (also known as MAPK13) in a model of LPS-induced hepatitis was shown to stimulate eEF2 activity in macrophages ${ }^{114}$. As a consequence, the translation of TNF is upregulated, which induces apoptosis and necrosis of hepatic cells. Interestingly, partial knockdown of eEF2 using small interfering RNAs blocked TNF expression by macrophages following LPS stimulation both in vitro and in vivo, and this blockade was sufficient to protect mice from liver failure. This result highlights the importance of the regulation of translation elongation in pro-inflammatory cytokine expression. As regulation of eEF2 activity should have an impact on the translation of most cellular mRNAs, it would be of interest to monitor its effect on additional cellular functions.

\section{IncRNAs in innate immunity}

Although miRNAs modulate inflammatory gene expression ${ }^{62,63}$, exciting recent studies support important roles for additional non-coding RNAs in this setting. Although several lncRNAs were discovered and characterized prior to 2005 (REFS 115-117), advances in sequencing and array technologies over the past few years have led to the discovery of thousands of lncRNA 


\section{Box 1 | Emerging roles of long non-coding RNAs in immunity}

Recent studies have revealed functional roles for long non-coding RNAs (IncRNAs) in immunity. The IncRNA Tmevpg1 (also known as NeST) controls Theiler's virus persistence in mice ${ }^{126,127}$ by promoting the transcription of interferon- $\gamma$ (Ifng) in CD8 ${ }^{+} T$ cells. The Tmevpg1 lncRNA binds to WD repeat-containing protein 5 (WDR5), a histone-modifying complex, altering histone $3(\mathrm{H} 3)$ lysine 4 trimethylation at the Ifng locus. Studies in macrophages have also revealed important roles for IncRNAs in controlling inflammatory gene expression. Many lncRNAs were found to be dynamically regulated in macrophages that were exposed to Toll-like receptor 2 (TLR2) ligands (see figure). One such transcript, long intergenic non-coding RNA (lincRNA)-Cox2, was found to act as a master regulator of gene expression. lincRNA-Cox2 represses the basal expression of interferon-stimulated genes (ISGs) by partnering with the heterogeneous nuclear ribonucleoproteins (HNRNPs) HNRNPA/B and HNRNPA2/B1. Remarkably, lincRNA-Cox2 was also essential for the TLR-induced expression of interleukin-6 (Il6) and more than 700 additional genes - many of which are secondary response genes ${ }^{128}$ — through a mechanism that remains to be fully elucidated (indicated by a question mark in the figure). A pseudogene RNA named Lethe (also known as Rps15a-ps4) binds RELA — the 065 subunit of the nuclear factor- $\kappa B$ (NF- $\kappa B$ ) heterodimeric complex - which prevents NF- $\mathrm{kB}$ from binding to promoter regions of target genes ${ }^{129}$. Finally, a lincRNA called TNF and HNRNPL-related immunoregulatory lincRNA (THRIL) was shown to regulate the expression of tumour necrosis factor (TNF) in human monocytes through its interactions with HNRNPL ${ }^{130}$. Collectively, these studies emphasize the importance of IncRNAs in regulating gene expression in macrophages and highlight yet another layer of complexity in gene regulation. Further analysis of their molecular functions could provide important insights into gene regulation, inflammation and human diseases.

IncRNAs can also act via post-transcriptional mechanisms altering mRNA splicing, turnover or translation. IncRNAs can act as microRNA (miRNA) sponges by preventing miRNA-mediated degradation of target mRNAs ${ }^{131}$. Metastasisassociated lung adenocarcinoma transcript 1 (MALAT1) controls alternative splicing of mRNA, whereas a newly defined class of IncRNAs (that is referred to as sno-lincRNAs) can affect RNA-binding protein fox-1 homologue 2 (FOX2)-mediated pre-mRNA splicing ${ }^{132,133}$. The IncRNA $\beta$-secretase 1 antisense transcript (BACE1-AS), which is upregulated in the brains of patients with Alzheimer's disease, stabilizes its protein-coding sense transcript BACE1 by protecting it from RNase cleavage ${ }^{134}$. Hu-antigen R (HUR) can drive the translation of several mRNAs in a IncRNA-dependent manner. In HeLa cells, lincRNA-p21 (also known as Trp53cor1) can interact with several mRNAs through direct base-pairing at complementary regions, repressing translation in a mechanism that requires DEAD-box protein 6 (DDX6) ${ }^{135}$. The role of IncRNAs in posttranscriptional gene regulation has been reviewed extensively ${ }^{136}$. Whether IncRNAs control gene expression through these mechanisms in the context of innate immune signalling remains to be determined.

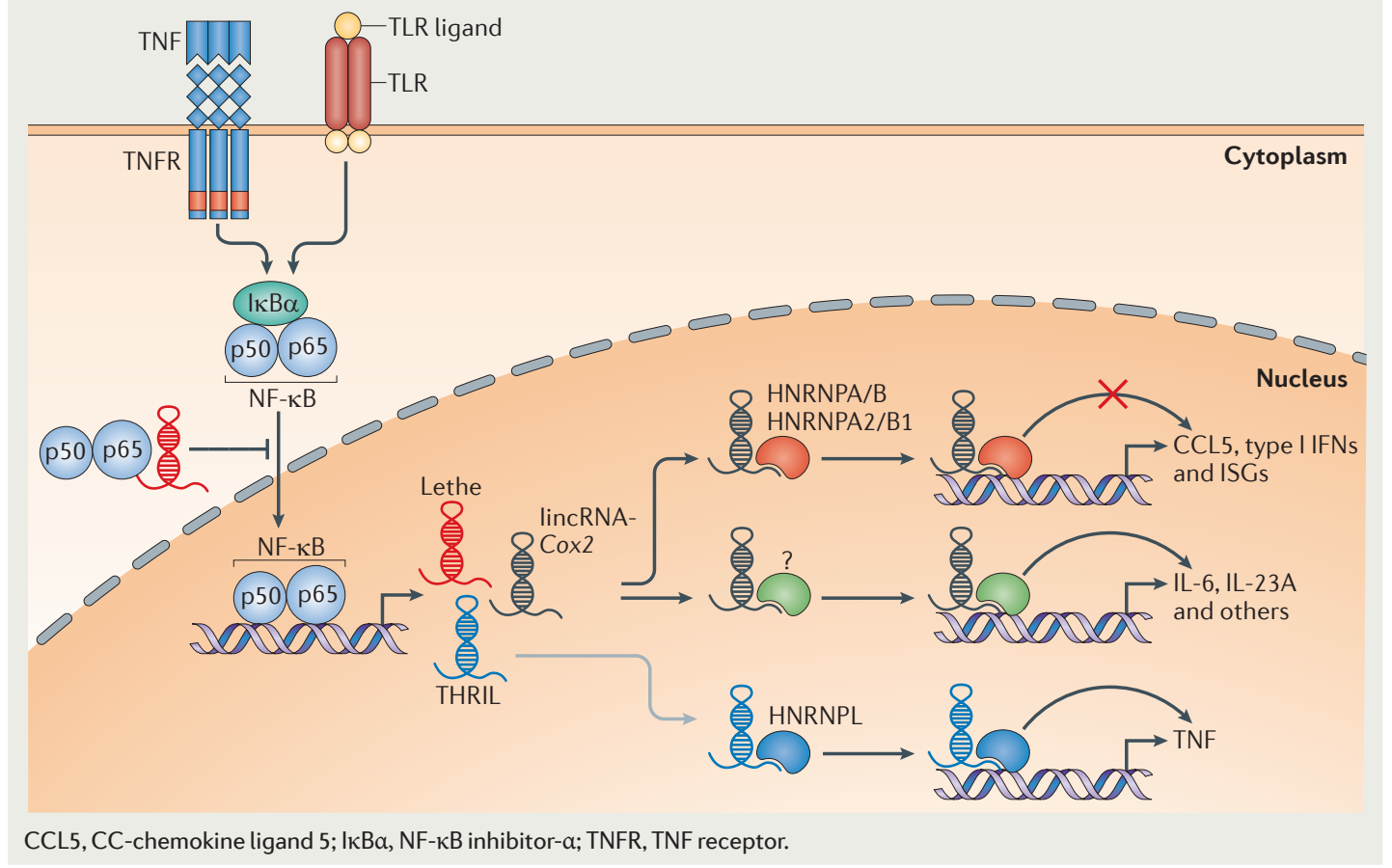

transcripts in diverse cell types ${ }^{118-124}$. These lncRNAs have primarily been studied in the context of genomic imprinting, cancer and cell differentiation. More recently, however, their expression in immune cells has prompted investigation into their roles in transcriptional and post-transcriptional regulation of immune gene expression (BOX 1).

\section{Conclusions and perspectives}

This Review highlights the wealth of post-transcriptional mechanisms that control the expression levels of immune genes. Although transcriptional regulation has been the focus in this area, it is clear that splicing, polyadenylation, mRNA stability and protein translation all act in concert to fine-tune and modulate the initiation, 
duration and magnitude of inflammatory gene expression in innate immunity. The expression of inhibitory splice variants that are induced by inflammatory signals, as well as tight control of mRNA half-lives, enable rapid and transient responses. Furthermore, regulation of mRNA translation allows a rapid response that can be directed against a specific set of genes or against the entire transcript population. Although exuberant 'on' signals clearly contribute to chronic inflammation, dysregulation of the 'off' signals can be equally damaging to tissues. Turning off the system in a timely and efficient manner is essential. The existence of multiple and apparently non-redundant regulatory mechanisms raises an important question concerning the relative importance of these individual controls. Such control at multiple checkpoints suggests that, individually, these hurdles are not sufficient to modulate a particular response, and a concerted effort by multiple regulatory mechanisms is required. A broader understanding of all of the layers of regulation in this system can provide important information that could be harnessed in vaccine development to improve the efficacy and duration of vaccineinduced immunity. Additionally, these multiple layers could be modulated therapeutically to thwart chronic inflammation, which contributes to a growing array of human diseases.
1. Aringer, M., Gunther, C. \& Lee-Kirsch, M. A. Innate immune processes in lupus erythematosus. Clin Immunol. 147, 216-222 (2013).

2. Moore, K. J., Sheedy, F. J. \& Fisher, E. A. Macrophages in atherosclerosis: a dynamic balance. Nature Rev. Immunol. 13, 709-721 (2013).

3. Kumar, H., Kawai, T. \& Akira, S. Pathogen recognition by the innate immune system. Int. Rev. Immunol. 30 , 16-34 (2011)

4. Thompson, M. R., Kaminski, J. J., Kurt-Jones, E. A. \& Fitzgerald, K. A. Pattern recognition receptors and the innate immune response to viral infection. Viruses 3 . 920-940 (2011)

5. Medzhitov, R. \& Horng, T. Transcriptional control of the inflammatory response. Nature Rev. Immunol. 9, 692-703 (2009)

6. Smale, S. T. Transcriptional regulation in the innate immune system. Curr. Opin. Immunol. 24, 51-57 (2012).

7. Rodrigues, R., Grosso, A. R. \& Moita, L. Genome-wide analysis of alternative splicing during dendritic cell response to a bacterial challenge. PLOS ONE 8 e61975 (2013).

8. Sandberg, R., Neilson, J. R., Sarma, A., Sharp, P. A. \& Burge, C. B. Proliferating cells express mRNAs with shortened 3' untranslated regions and fewer microRNA target sites. Science 320, 1643-1647 (2008).

9. Moore, M. J. \& Proudfoot, N. J. Pre-mRNA processing reaches back to transcription and ahead to translation. Cell 136, 688-700 (2009).

10. Elkon, R., Ugalde, A. P. \& Agami, R. Alternative cleavage and polyadenylation: extent, regulation and function. Nature Rev. Genet. 14, 496-506 (2013).

11. Wang, E. T. et al. Alternative isoform regulation in human tissue transcriptomes. Nature 456, 470-476 (2008).

12. Miura, P., Shenker, S., Andreu-Agullo, C., Westholm, J. O. \& Lai, E. C. Widespread and extensive lengthening of 3' UTRs in the mammalian brain. Genome Res. 23, 812-825 (2013)

13. Wells, C. A. et al. Alternate transcription of the Toll-like receptor signaling cascade. Genome Biol. 7, R10 (2006).

14. Jarešová, I. et al. Kinetics of Toll-like receptor-4 splice variants expression in lipopolysaccharide-stimulated antigen presenting cells of healthy donors and patients with cystic fibrosis. Microbes Infect. 9, 1359-1367 (2007).

15. Iwami, K. I. et al. Cutting edge: naturally occurring soluble form of mouse Toll-like receptor 4 inhibits lipopolysaccharide signaling. J. Immunol. 165 6682-6686 (2000).

16. Stevenson, B. J., Iseli, C., Beutler, B. \& Jongeneel, C. V. Use of transcriptome data to unravel the fine structure of genes involved in sepsis. J. Infect. Dis. 187 (Suppl. 2), 308-314 (2003).

17. Chang, J.-S. et al. Myobacterium tuberculosis induces selective up-regulation of TLRs in the mononuclear leukocytes of patients with active pulmonary tuberculosis. J. Immunol. 176, 3010-3018 (2006).

18. Haehnel, V., Schwarzfischer, L., Fenton, M. J. \& Rehli, M. Transcriptional regulation of the human Tolllike receptor 2 gene in monocytes and macrophages. J. Immunol. 168, 5629-5637 (2002).

19. Ohta, S., Bahrun, U., Tanaka, M. \& Kimoto, M Identification of a novel isoform of MD-2 that downregulates lipopolysaccharide signaling. Biochem Biophys. Res. Commun. 323, 1103-1108 (2004)
20. Gray, P. et al. Identification of a novel human MD-2 splice variant that negatively regulates lipopolysaccharide-induced TLR4 signaling. J. Immunol. 184, 6359-6366 (2010).

21. Burns, K. et al. Inhibition of interleukin 1 receptor/ Toll-like receptor signaling through the alternatively spliced, short form of MyD88 is due to its failure to recruit IRAK-4. J. Exp. Med. 197, 263-268 (2003)

This paper identifies MYD88s, which is a short dominant-negative inhibitor that is a splice variant of MYD88.

22. Janssens, S., Burns, K., Tschopp, J. \& Beyaert, R. Regulation of interleukin-1 - and lipopolysaccharideinduced NF- $\mathrm{kB}$ activation by alternative splicing of MyD88. Curr. Biol. 12, 467-471 (2002).

23. Janssens, S., Burns, K., Vercammen, E., Tschopp, J. \& Beyaert, R. MyD88S, a splice variant of MyD88, differentially modulates NF-kB-and AP-1-dependent gene expression. FEBS Lett. 548, 103-107 (2003).

24. Jensen, L. E. \& Whitehead, A. S. IRAK1b, a novel alternative splice variant of interleukin-1 receptorassociated kinase (IRAK), mediates interleukin-1 signaling and has prolonged stability. J. Biol. Chem 276, 29037-29044 (2001).

25. Yanagisawa, K. et al. A novel splice variant of mouse interleukin-1-receptor-associated kinase-1 (IRAK-1)

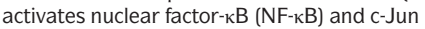
$\mathrm{N}$-terminal kinase (JNK). Biochem. J. 370, 159-166 (2003)

26. Rao, N., Nguyen, S., Ngo, K. \& Fung-Leung, W.-P. A novel splice variant of interleukin-1 receptor (IL-1R)associated kinase 1 plays a negative regulatory role in Toll/IL-1 R-induced inflammatory signaling. Mol. Cell. Biol. 25, 6521-6532 (2005)

27. Hardy, M. P. \& O'Neill, L. A. J. The murine IRAK2 gene encodes four alternatively spliced isoforms, two of which are inhibitory. J. Biol. Chem. 279 27699-27708 (2004)

28. Leeman, J. R. \& Gilmore, T. D. Alternative splicing in the NF-кB signaling pathway. Gene 423, 97-107 (2008).

29. Han, K.-J., Yang, Y., Xu, L.-G. \& Shu, H.-B. Analysis of a TIR-less splice variant of TRIF reveals an unexpected mechanism of TLR3-mediated signaling. J. Biol. Chem. 285, 12543-12550 (2010).

30. Palsson-McDermott, E. M. et al. TAG, a splice variant of the adaptor TRAM, negatively regulates the adaptor MyD88-independent TLR4 pathway. Nature Immunol. 10, 579-586 (2009). This paper identifies TAG, which is an inhibitory splice variant of the adaptor molecule TRAM.

31. Karpova, A. Y., Ronco, L. V. \& Howley, P. M Functional characterization of interferon regulatory factor 3a (IRF-3a), an alternative splice isoform of IRF-3. Mol. Cell. Biol. 21, 4169-4176 (2001).

32. Li, C., Ma, L. \& Chen, $X$. Interferon regulatory factor 3-CL, an isoform of IRF3, antagonizes activity of IRF3 Cell. Mol. Immunol. 8, 67-74 (2011)

33. Li, Y. et al. Identification of novel alternative splicing variants of interferon regulatory factor 3 . Biochim. Biophys. Acta 1809, 166-175 (2011).

34. Shell, S. A., Hesse, C., Morris, S. M. \& Milcarek, C. Elevated levels of the $64-\mathrm{kDa}$ cleavage stimulatory factor (CstF-64) in lipopolysaccharide-stimulated macrophages influence gene expression and induce alternative poly(A) site selection. J. Biol. Chem. 280 39950-39961 (2005).
35. Bhatt, D. M. et al. Transcript dynamics of proinflammatory genes revealed by sequence analysis of subcellular RNA fractions. Cell 150, 279-290 (2012). This paper indicates that the kinetics of pre-mRNA splicing regulate the temporal order of pro-inflammatory gene expression.

36. Hao, S. \& Baltimore, D. RNA splicing regulates the temporal order of TNF-induced gene expression. Proc. Natl Acad. Sci. USA 110, 11934-11939 (2013)

37. Rabani, M. et al. Metabolic labeling of RNA uncovers principles of RNA production and degradation dynamics in mammalian cells. Nature Biotech. 29, 436-442 (2011)

This paper analyses the respective contributions of gene transcription and RNA decay to the responses of DCs to LPS.

38. Paulsen, M. T. et al. Coordinated regulation of synthesis and stability of RNA during the acute TNFinduced proinflammatory response. Proc. Natl Acad. Sci. USA 110, 2240-2245 (2013).

This paper analyses the respective contributions of gene transcription and RNA degradation to TNF-induced cell responses.

39. Elkon, R., Zlotorynski, E., Zeller, K. I. \& Agami, R. Major role for mRNA stability in shaping the kinetics of gene induction. BMC Genomics 11, 259 (2010).

40. Hao, S. \& Baltimore, D. The stability of mRNA influences the temporal order of the induction of genes encoding inflammatory molecules. Nature Immunol. 10, 281-288 (2009).

41. Caput, D. et al Identification of a common nucleotide sequence in the $3^{\prime}$ untranslated region of mRNA molecules specifying inflammatory mediators. Proc. Natl Acad. Sci. USA 83, 1670-1674 (1986). This paper is the first to identify an ARE in TNF transcripts and its role in mRNA degradation.

42. Shaw, G. \& Kamen, R. A conserved AU sequence from the 3' untranslated region of GM-CSF mRNA mediates selective mRNA degradation. Cell 46, 659-667 (1986).

This paper is the first to identify an ARE in CSF2 transcripts and its role in mRNA degradation.

43. Bakheet, T., Williams, B. R. \& Khabar, K. S. ARED 3.0: the large and diverse AU-rich transcriptome. Nucleic Acids Res. 34, D111-114 (2006).

44. Halees, A. S., El-Badrawi, R. \& Khabar, K. S. ARED Organism: expansion of ARED reveals AU-rich element cluster variations between human and mouse. Nucleic Acids Res. 36, D137-D140 (2008).

45. Beisang, D. \& Bohjanen, P. R. Perspectives on the ARE as it turns 25 years old. Wiley Interdiscip. Rev. RNA 3 719-731 (2012)

46. Ivanov, P. \& Anderson, P. Post-transcriptional regulatory networks in immunity. Immunol. Rev. 253 , 253-272 (2013).

47. Kontoyiannis, D., Pasparakis, M., Pizarro, T. T. Cominelli, F. \& Kollias, G. Impaired on/off regulation of TNF biosynthesis in mice lacking TNF AU-rich elements: implications for joint and gut-associated immunopathologies. Immunity 10, 387-398 (1999).

48. Chen, Y.-L. et al. Differential regulation of AREmediated TNF $\alpha$ and IL- $1 \beta$ mRNA stability by lipopolysaccharide in RAW264.7 cells. Biochem Biophys. Res. Commun. 346, 160-168 (2006)

49. Stoecklin, G. et al. MK2-induced tristetraprolin:14-3-3 complexes prevent stress granule association and ARE-mRNA decay. EMBO J. 23, 1313-1324 (2004). 
50. Sun, L. et al. Tristetraprolin (TTP)-14-3-3 complex formation protects TTP from dephosphorylation by protein phosphatase $2 \mathrm{a}$ and stabilizes tumor necrosis factor- $\alpha$ mRNA. J. Biol. Chem. 282, 3766-3777 (2007).

51. Lu, J.-Y., Sadri, N. \& Schneider, R. J. Endotoxic shock in AUF1 knockout mice mediated by failure to degrade proinflammatory cytokine mRNAs. Genes Dev. 20, 3174-3184 (2006).

52. Taylor, G. A. et al. A pathogenetic role for TNFa in the syndrome of cachexia, arthritis, and autoimmunity resulting from tristetraprolin (TTP) deficiency. Immunity 4, 445-454 (1996).

53. Carballo, E., Lai, W. S. \& Blackshear, P. J. Evidence that tristetraprolin is a physiological regulator of granulocyte-macrophage colony-stimulating factor messenger RNA deadenylation and stability. Blood 95, 1891-1899 (2000)

54. Sadri, N. \& Schneider, R. J. Auf1/Hnrnpd-deficient mice develop pruritic inflammatory skin disease. J. Invest. Dermatol. 129, 657-670 (2009).

55. Yiakouvaki, A. et al. Myeloid cell expression of the RNA-binding protein HuR protects mice from pathologic inflammation and colorectal carcinogenesis. J. Clin. Invest. 122, 48-61 (2012).

56. Stoecklin, G., Lu, M., Rattenbacher, B. \& Moroni, C. A constitutive decay element promotes tumor necrosis factor alpha mRNA degradation via an AU-rich element-independent pathway. Mol. Cell. Biol. 23. 3506-3515 (2003).

57. Moraes, K. C. M., Wilusz, C. J. \& Wilusz, J. CUG-BP binds to RNA substrates and recruits PARN deadenylase. RNA 12, 1084-1091 (2006).

58. Pautz, A. et al. The polypyrimidine tract-binding protein (PTB) is involved in the post-transcriptional regulation of human inducible nitric oxide synthase expression. J. Biol. Chem. 281, 32294-32302 (2006).

59. Fabian, M. R. \& Sonenberg, N. The mechanics of miRNA-mediated gene silencing: a look under the hood of miRISC. Nature Struct. Mol. Biol. 19 586-593 (2012)

60. Kozomara, A. \& Griffiths-Jones, S. miRBase: integrating microRNA annotation and deepsequencing data. Nucleic Acids Res. 39, D152-D157 (2011).

61. Friedman, R. C., Farh, K. K.-H., Burge, C. B. \& Bartel, D. P. Most mammalian mRNAs are conserved targets of microRNAs. Genome Res. 19, 92-105 (2009).

62. O'Connell, R. M. \& Rao, D. S. \& Baltimore, D microRNA regulation of inflammatory responses. Annu. Rev. Immunol. 30, 295-312 (2012).

63. Li, Y. \& Shi, X. MicroRNAs in the regulation of TLR and RIG-I pathways. Cell. Mol. Immunol. 10, 65-71 (2013).

64. Bicknell, A. A., Cenik, C., Chua, H. N., Roth, F. P. \& Moore, M. J. Introns in UTRs: why we should stop ignoring them. Bioessays 34, 1025-1034 (2012).

65. Weischenfeldt, J et al. NMD is essential for hematopoietic stem and progenitor cells and for eliminating by-products of programmed DNA rearrangements. Genes Dev. 22, 1381-1396 (2008). This paper indicates a role for NMD in gene expression regulation in macrophages.

66. Lelouard, H. et al. Regulation of translation is required for dendritic cell function and survival during activation. J. Cell Biol. 179, 1427-1439 (2007).

67. Donnelly, N., Gorman, A. M., Gupta, S. \& Samali, A The elF2 $\alpha$ kinases: their structures and functions. Cell. Mol. Life Sci. 70, 3493-3511 (2013)

68. He, B. Viruses, endoplasmic reticulum stress, and interferon responses. Cell Death Differ. 13, 393-403 (2006).

69. Woo, C. W. et al. Adaptive suppression of the ATF4-CHOP branch of the unfolded protein response by toll-like receptor signalling. Nature Cell Biol. 11, 1473-1480 (2009).

70. Woo, C. W., Kutzler, L., Kimball, S. R. \& Tabas, I. Toll-like receptor activation suppresses ER stress factor $\mathrm{CHOP}$ and translation inhibition through activation of elF2B. Nature Cell Biol. 14, 192-200 (2012).

This paper shows that in ER-stressed macrophages the activity of eIF2B is stimulated to overcome translational repression caused by eIF $2 \alpha$ phosphorylation.

71. Furic, L. et al. elF4E phosphorylation promotes tumorigenesis and is associated with prostate cancer progression. Proc. Natl Acad. Sci. USA 107 14134-14139 (2010).
72. Kleijn, M., Vrins, C. L., Voorma, H. O. \& Thomas, A. A Phosphorylation state of the cap-binding protein eIF4E during viral infection. Virology 217, 486-494 (1996).

73. Herdy, B et al. Translational control of the activation of transcription factor NF-kB and production of type interferon by phosphorylation of the translation factor elF4E. Nature Immunol. 13, 543-550 (2012) This paper shows that eIF4E phosphorylation levels regulate the translation of I $\mathrm{kB} \alpha$ to modulate type I IFN responses.

74. Wan, Y. et al. Interleukin-1 receptor-associated kinase 2 is critical for lipopolysaccharide-mediated post-transcriptional control. J. Biol. Chem. 284 10367-10375 (2009)

75. Zhou, H. et al. IRAK-M mediates Toll-like receptor IL-1 R-induced $\mathrm{NF}_{\mathrm{KB}} \mathrm{B}$ activation and cytokine production. EMBO J. 32, 583-596 (2013)

76. Kim, W. J., Kim, J. H. \& Jang, S. K. Anti-inflammatory lipid mediator 15d-PGJ2 inhibits translation through inactivation of elF4A. EMBO J. 26, 5020-5032 (2007)

77. Schmitz, F. et al. Mammalian target of rapamycin (mTOR) orchestrates the defense program of innate immune cells. Eur. J. Immunol. 38, 2981-2992 (2008)

78. Colina, R. et al. Translational control of the innate immune response through IRF-7. Nature 452 323-328 (2008)

This paper shows that $4 \mathrm{EBP}$-deficient mice have increased type I IFN responses and are less susceptible to viral infection.

79. López-Pelaéz, M. et al. Cot/tpl2-MKK1/2-Erk1/2 controls mTORC1-mediated mRNA translation in Toll-like receptor-activated macrophages. Mol. Biol. Cell 23, 2982-2992 (2012).

This paper shows that 4EBP1 phosphorylation mediated by MAP3K8 (referred to as Cot/tpl2 above) upon TLR engagement stimulates cap-dependent translation.

80. Jefferies, H. B. et al. Rapamycin suppresses 5'TOP mRNA translation through inhibition of p70s6k. EMBO J. 16, 3693-3704 (1997)

81. Jaramillo, M. et al. Leishmania repression of host translation through mTOR cleavage is required for parasite survival and infection. Cell Host Microbe 9, 331-341 (2011).

82. Weichhart, T. et al. The TSC-mTOR signaling pathway regulates the innate inflammatory response. Immunity 29, 565-577 (2008).

83. Ivanov, S. S. \& Roy, C. R. Pathogen signatures activate a ubiquitination pathway that modulates the function of the metabolic checkpoint kinase mTOR Nature Immunol. 14, 1219-1228 (2013).

84. Jackson, R. J., Hellen, C. U. T. \& Pestova, T. V. The mechanism of eukaryotic translation initiation and principles of its regulation. Nature Rev. Mol. Cell. Biol. 11, 113-127 (2010).

85. Weill, L., Belloc, E., Bava, F.-A. \& Méndez, R. Translational control by changes in poly(A) tail length recycling mRNAs. Nature Struct. Mol. Biol. 19, 577-585 (2012)

86. Crawford, E. K., Ensor, J. E., Kalvakolanu, I. \& Hasday, J. D. The role of 3' poly(A) tail metabolism in tumor necrosis factor- $\alpha$ regulation. J. Biol. Chem 272 21120-21127 (1997)

87. Swanson, B. J., Murakami, M., Mitchell, T. C., Kappler, J. \& Marrack, P. RANTES production by memory phenotype T cells is controlled by a posttranscriptional, TCR-dependent process. Immunity 17, 605-615 (2002).

88. Groppo, R. \& Richter, J. D. CPEB control of NF-кB nuclear localization and interleukin-6 production mediates cellular senescence. Mol. Cell. Biol. 31 2707-2714 (2011).

89. Kozak, M. An analysis of 5'-noncoding sequences from 699 vertebrate messenger RNAs. Nucleic Acids Res. 15, 8125-8148 (1987)

90. Shinohara, M. L., Kim, H.-J., Kim, J.-H., Garcia, V. A. \& Cantor, H. Alternative translation of osteopontin generates intracellular and secreted isoforms that mediate distinct biological activities in dendritic cells. Proc. Natl Acad. Sci. USA 105, 7235-7239 (2008)

91. Kozak, M. Constraints on reinitiation of translation in mammals. Nucleic Acids Res. 29, 5226-5232 (2001)

92. Calvo, S. E., Pagliarini, D. J. \& Mootha, V. K. Upstream open reading frames cause widespread reduction of protein expression and are polymorphic among humans. Proc. Natl Acad. Sci. USA 106, 7507-7512 (2009).
93. Brubaker, S. W., Gauthier, A. E., Mills, E. W. Ingolia, N. T. \& Kagan, J. C. A bicistronic MAVS transcript highlights a class of truncated variants in antiviral immunity. Cell 156, 800-811 (2014). This paper shows that two isoforms of MAVS with antagonistic functions are produced from a single transcript through leaky scanning and translation re-initiation.

94. Lang, K. J. D., Kappel, A. \& Goodall, G. J. Hypoxiainducible factor- 1 a mRNA contains an internal ribosome entry site that allows efficient translation during normoxia and hypoxia. Mol. Biol. Cell 13, 1792-1801 (2002).

95. Wang, G., Guo, X., Silveyra, P., Kimball, S. R. \& Floros, J. Cap-independent translation of human SP-A 5'-UTR variants: a double-loop structure and ciselement contribution. Am. J. Physiol. Lung Cell. Mol. Physiol. 296, L635-L647 (2009).

96. Rübsamen, D. et al. IRES-dependent translation of egr2 is induced under inflammatory conditions. RNA 18, 1910-1920 (2012).

97. Rübsamen, D. et al. Inflammatory conditions induce IRES-dependent translation of cyp24a1. PLOS ONE 9, e85314 (2014).

98. Leppek, K. \& Stoecklin, G. An optimized streptavidinbinding RNA aptamer for purification of ribonucleoprotein complexes identifies novel AREbinding proteins. Nucleic Acids Res. 42, e 13 (2013)

99. Tiedje, C. et al. The p38/MK2-driven exchange between tristetraprolin and HuR regulates AU-rich element-dependent translation. PLoS Genet. 8 e1002977 (2012).

100. Qi, M.-Y. et al. AU-rich-element-dependent translation repression requires the cooperation of tristetraprolin and RCK/P54. Mol. Cell. Biol. 32, 913-928 (2012).

101. Piecyk, M. et al. TIA-1 is a translational silencer that selectively regulates the expression of TNF- $\alpha$. EMBO J. 19, 4154-4163 (2000).

102. Anderson, P. \& Kedersha, N. Visibly stressed: the role of elF2, TIA-1, and stress granules in protein translation. Cell Stress Chaperones 7, 213-221 (2002).

103. Damgaard, C. K. \& Lykke-Andersen, J. Translational coregulation of 5 ' TOP mRNAs by TIA-1 and TIAR. Genes Dev. 25, 2057-2068 (2011)

104. Mazumder, B. \& Fox, P. L. Delayed translational silencing of ceruloplasmin transcript in gamma interferon-activated U937 monocytic cells: role of the 3 ' untranslated region. Mol. Cell. Biol. 19, 68986905 (1999).

105. Sampath, P., Mazumder, B., Seshadri, V. \& Fox, P. L. Transcript-selective translational silencing by gamma interferon is directed by a novel structural element in the ceruloplasmin mRNA 3' untranslated region. Mol. Cell. Biol. 23, 1509-1519 (2003).

106. Sampath, P. et al. Noncanonical function of glutamylprolyl-tRNA synthetase: gene-specific silencing of translation. Cell 119, 195-208 (2004).

This paper describes the sequential assembly of the GAIT complex and the binding of the complex to its target mRNA upon IFN $\gamma$ exposure.

107. Mazumder, B. et al. Regulated release of $L 13$ a from the $60 \mathrm{~S}$ ribosomal subunit as a mechanism of transcript-specific translational control. Cell 115 , 187-198 (2003)

108. Kapasi, P. et al. L13a blocks 48 S assembly: role of a general initiation factor in mRNA-specific translationa control. Mol. Cell 25, 113-126 (2007).

109. Vyas, K. et al. Genome-wide polysome profiling reveals an inflammation-responsive posttranscriptional operon in gamma interferon-activated monocytes. Mol. Cell. Biol. 29, 458-470 (2008).

110. Mukhopadhyay, R. et al. DAPK-ZIPK-L13a axis constitutes a negative-feedback module regulating inflammatory gene expression. Mol. Cell 32, 371-382 (2008).

111. Ray, P. S. \& Fox, P. L. A post-transcriptional pathway represses monocyte VEGF-A expression and angiogenic activity. EMBO J. 26, 3360-3372 (2007).

112. Ray, P. S. et al. A stress-responsive RNA switch regulates VEGFA expression. Nature 457, 915-919 (2009).

13. Yao, P. et al. The HILDA complex coordinates a conditional switch in the 3'-untranslated region of the VEGFA mRNA. PLoS Biol. 11, e 1001635 (2013).

114. González-Terăn, B. et al. Eukaryotic elongation factor 2 controls TNF- $\alpha$ translation in LPS-induced hepatitis. J. Clin. Invest. 123, 164-178 (2013). This paper shows that LPS-induced MAPK activation stimulates eEF2 activity to increase TNF translation elongation. 
115. Pachnis, V., Brannan, C. I. \& Tilghman, S. M The structure and expression of a novel gene activated in early mouse embryogenesis. EMBO J. 7, 673-681 (1988).

116. Brannan, C. I., Dees, E. C., Ingram, R. S. \& Tilghman, S. M. The product of the $\mathrm{H} 19$ gene may function as an RNA. Mol. Cell. Biol. 10, 28-36 function

117. Penny, G. D., Kay, G. F., Sheardown, S. A., Rastan, S. \& Brockdorff, N. Requirement for Xist in X chromosome inactivation. Nature 379, 131-137 (1996).

118. Guttman, M. et al. Chromatin signature reveals over a thousand highly conserved large non-coding RNAs in mammals. Nature 458, 223-227 (2009).

119. Rinn, J. L. \& Chang, H. Y. Genome regulation by long noncoding RNAs. Annu. Rev. Biochem. 81, 145-166 (2012).

120. Mortazavi, A., Williams, B. A., McCue, K., Schaeffer, L. $£$ Wold, B. Mapping and quantifying mammalian transcriptomes by RNA-Seq. Nature Methods $\mathbf{5}$, 621-628 (2008)

121. Guttman, M. et al. Ab initio reconstruction of cell typespecific transcriptomes in mouse reveals the conserved multi-exonic structure of lincRNAs. Nature Biotech. 28, 503-510 (2010).

122. Guttman, M. \& Rinn, J. L. Modular regulatory principles of large non-coding RNAs. Nature 482, 339-346 (2012)
123. Birney, E. et al. Identification and analysis of functional elements in $1 \%$ of the human genome by the ENCODE pilot project. Nature 447, 799-816 (2007).

124. Peng, X. et al. Unique signatures of long noncoding RNA expression in response to virus infection and altered innate immune signaling. $\mathrm{mBio} 1$ e00206-e00210 (2010).

125. Ricci, E. P. et al. miRNA repression of translation in vitro takes place during $43 \mathrm{~S}$ ribosomal scanning. Nucleic Acids Res. 41, 586-598 (2013).

126. Gomez, J. A. et al. The NeST long ncRNA controls microbial susceptibility and epigenetic activation of the interferon- $\gamma$ locus. Cell 152, 743-754 (2013).

127. Collier, S. P., Collins, P. L., Williams, C. L., Boothby, M. R. \& Aune, T. M. Cutting edge: influence of Tmevpg 1, a long intergenic noncoding RNA, on the expression of Ifng by $\mathrm{T}_{\mathrm{H}} 1$ cells. J. Immunol. 189 2084-2088 (2012).

128. Carpenter, S. et al. A long noncoding RNA mediates both activation and repression of immune response genes. Science 341, 789-792 (2013).

129. Rapicavoli, N. A. et al. A mammalian pseudogene IncRNA at the interface of inflammation and antiinflammatory therapeutics. eLife 2, e00762 (2013).

130. Li, Z. et al. The long noncoding RNA THRIL regulates TNF $\alpha$ expression through its interaction with hnRNPL. Proc. Natl Acad. Sci. USA 111, 1002-1007 (2014).
131. Salmena, L., Poliseno, L., Tay, Y., Kats, L. \& Pandolfi, P. P. A ceRNA hypothesis: the Rosetta Stone of a hidden RNA language? Cell 146, 353-358 (2011).

132. Zong, X., Tripathi, V. \& Prasanth, K. V. RNA splicing control: yet another gene regulatory role for long nuclear noncoding RNAs. RNA Biol. 8, 968-977 nuclear
(2011).

133. Yin, Q. F. et al. Long noncoding RNAs with snoRNA ends. Mol. Cell 48, 219-230 (2012).

134. Faghihi, M. A. et al. Expression of a noncoding RNA is elevated in Alzheimer's disease and drives rapid feedforward regulation of $\beta$-secretase. Nature Med. 14 , 723-730 (2008)

135. Yoon, J.-H. et al. LincRNA-p21 suppresses target mRNA translation. Mol. Cell 47, 648-655 (2012).

136. Yoon, J.-H., Abdelmohsen, K. \& Gorospe, M Posttranscriptional gene regulation by long noncoding RNA. J. Mol. Biol. 425, 3723-3730 (2013).

Acknowledgements

This work is supported by the US National Institutes of Health (grant AI067497 to K.A.F) and the Howard Hughes Medical Institute, USA (M.J.M).

Competing interests statement

The authors declare no competing interests. 